\title{
Miltos Pechlivanos
}

\section{Der griechische Bürgerkrieg und das geteilte Berlin: „Weiße Rosen aus Athen“ und Antigone lebt}

\section{Der griechische „dreißigjährige Krieg“ und das deutsch-griechische Dreieck}

Die drei Jahrzehnte zwischen 1943, mit dem Anbruch der ersten innergriechischen, bürgerkriegsähnlichen Auseinandersetzungen zwischen links- und rechtsgerichteten Widerstandsorganisationen schon während der Zeit der deutschen Besatzung, und 1973, mit der blutigen Niederschlagung des Studierendenaufstandes von den Panzern im Polytechnikum, der Athener Technischen Universität, hat der Autor und Literaturkritiker Alexandros Kotzias (1924-1992) als den griechischen „dreißigjährigen Krieg“ bezeichnet. ${ }^{1}$ Die Pointe dieser zugespitzten Formulierung, die die tief spaltende Polarisierung, die gewaltsame und quasi konfessionelle Zerrissenheit der griechischen Nachkriegsgesellschaft zwischen rechts und links, Antikommunismus und kommunistischer Erlösung, unterstreicht, liegt auf dem ungebrochenen Fortwirken des Bürgerkriegserbes. $^{2}$

Eingebettet in den globalen Systemkonflikt, den sich anbahnenden Kalten Krieg, als dessen erster Stellvertreterkrieg der griechisch-griechische angesehen werden kann, warf der offene Bürgerkrieg der Jahre 1946-1949 seinen langen Schatten in die Zukunft bis zu den 1970er Jahren und schöpfte seine Narrative aus den Alltagserfahrungen

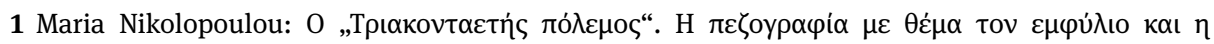

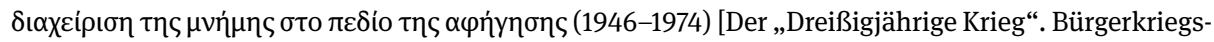
prosa und der Umgang mit der Erinnerung im Feld der Erzählung (1946-1974)], in: Giorgos Antoniou,

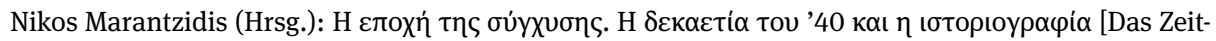
alter der Verwirrung. Die 1940er Jahre und die Historiographie], Athen 2008, 419-493. Zur Thematik der Bürgerkriegsrepräsentationen vgl. den Band: Athanasios Anastasiadis, Ulrich Moennig (Hrsg.): Trauma und Erinnerung. Narrative Versionen zum Bürgerkrieg in Griechenland, Köln/Weimar/Wien 2018. Dass diese Bezeichnung von Kotzias zugleich mit einer selbstreflexiven Geste den Krieg als beendet postuliert, liegt auf der Hand; zu diesem langjährigen Prozess der Literatur und Literaturkritik vgl. Joachim Winkler: Dimitris Raftopoulos und die „Schwarze Literatur“. Zum erinnerungskulturellen Widerstands- und Bürgerkriegsdiskurs der griechischen Nachkriegsliteratur, Berlin 2018.

2 Siehe dazu den Überblick in den Kapiteln „Der Zweite Weltkrieg und seine Folgen (1940-1949)“ und „Vom Bürgerkrieg zur Diktatur (1950-1974)“ in Ioannis Zelepos: Kleine Geschichte Griechenlands: von der Staatsgründung bis heute, München 2014, 152-209, und vgl. Richard Clogg: Eine kurze Geschichte Griechenlands, übers. von Karin E. Seifert und Diana Siebert, Berlin 2020, Kap. 4 und 5.
} 
von Besatzung, Not und Gewalt, von Widerstand und Kollaboration bis zum Abzug der deutschen Truppen aus Griechenland im Oktober 1944; aus den wilden Straßenschlachten im Dezember 1944 in Athen zwischen britischen Streitkräften und Einheiten der Griechischen Volksbefreiungsarmee und dem „weißen Terror“ nach der Entwaffnung der Partisanen; aus den Deportationen der Sympathisanten der Linken auf Exilinseln und der Formierung eines rechtsextremen „Schattenstaates“ (Parallel-, Neben- oder Parastaates) unter Beteiligung ehemaliger Kollaborateure. Trotz ephemerer Ansätze zur Aussöhnung der Sieger mit den im Land verbliebenen Verlierern ist die brutale Rache tonangebend gewesen: Exekutionen und jahrelange Haft auf den Internierungsinseln, Stigmatisierung der Unterlegenen als Landesverräter und Beschwörung der antikommunistischen „Nationalgesinnung“ oder, wie die Obristen es auf den Punkt gebracht haben, des „Hellas hellenischer Christen“. Das ausgeprägte Spitzelwesen hat sich auch in Griechenland in den berüchtigten persönlichen „Dossiers sozialer Gesinnung“ niedergeschlagen, „die als Entscheidungsgrundlage nicht nur für die Einstellung in den öffentlichen Dienst, sondern auch für Anträge, etwa auf Reisepässe, Führerscheine etc. dienten [...] und bis zum Ende der Militärdiktatur 1974 ein Alltagsphänomen bleiben“ sollten. ${ }^{3}$ Und last but not least: Nach der Niederlage der kommunistischen Armee in den Gebirgen von Grammos und Vitsi im Sommer 1949 zogen sich zwischen 70000 und 100000 Männer, Frauen und Kinder über die albanische Grenze zurück und wurden als politische Flüchtlinge auf die UdSSR, Polen, Tschechoslowakei, Rumänien, Ungarn und die DDR verteilt, wo sie bis zu ihrer Repatriierung in den 1970er und 1980er Jahren lebten. ${ }^{4}$

Das so verstandene späte Ende des Bürgerkrieges, ${ }^{5}$ unter dem Vorzeichen des sich verschärfenden weltweiten Kalten Krieges, hat ebenfalls nachhaltig die trilateralen

3 Zelepos (Anm. 2), 185. Zu der Vernichtung von 17500000 Dossiers im August 1989, kurz vor dem Mauerfall, im Namen einer Versöhnung, die auf dem Vergessen beruhen solle, vgl. Vangelis Karama-

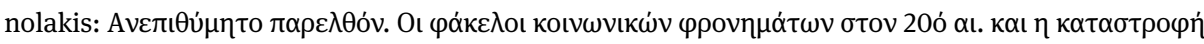
tovৎ [Unerwünschte Vergangenheit. Die Dossiers sozialer Gesinnung im 20. Jahrhundert und ihre Vernichtung], Athen 2019.

4 Adamantios Theodor Skordos: Das späte Ende des Bürgerkrieges. Die Diktatur der Obristen und deren Überwindung als politische Zäsur in der griechischen Geschichte des 20. Jahrhunderts, in: Jörg Ganzenmüller (Hrsg.): Europas vergessene Diktaturen? Diktatur und Diktaturüberwindung in Spanien, Portugal und Griechenland, Köln/Weimar/Wien 2018, 155-178, hier: 164. Vgl. dazu die Beiträge von Patrice G. Poutrus: Zwischen Internationalismus und Assimilation. Griechische „polit. Emigranten“ in der DDR, und von Maria Panoussi: Die Erziehung und Bildung der heranwachsenden Griechen nach ihrer Aufnahme in den 1950er Jahren in den Einrichtungen der DDR. Die staatlichen Erziehungsvorstellungen und die damit verbundenen Ziele, in: Marco Hillemann, Miltos Pechlivanos (Hrsg.): Deutsch-griechische Beziehungen im ostdeutschen Staatssozialismus (1949-1989). Politische Migration, Realpolitik und interkulturelle Begegnung, Berlin 2017, 61-75 und 77-91.

5 „An solch einer Perspektive vermag auch die zunehmende Öffnung der griechischen Gesellschaft nichts zu ändern, die mit gehäuft auftretenden Unruhen in den 1960er Jahren einherging. Paradigmatisch lässt sich [...] die weithin bekannte Ermordung des linken Politikers Grigoris Lamprakis im Jahr 
deutsch-griechischen Nachkriegsbeziehungen geprägt, die Selbst- und Fremdwahrnehmungen, die Freund- und Feindbilder im geteilten Griechenland und im geteilten Berlin bzw. zwischen Ostberlin und Bonn. Im deutsch-griechischen Dreieck, so Hagen Fleischer, demonstrierte die DDR stolz ihre antifaschistische Unschuld, etwa wenn DDRVertreter ,als erste Deutsche 1959 in Distomo und Chortiatis vielbeachtete Kränze zum ehrenden Gedenken der 1944 auf brutalste Weise massakrierten Einwohner“ niederlegten, jedoch hatte die DDR 1949/50 bei der Aufteilung der Exilierten nach dem Bürgerkrieg 1128 Kinder aus kommunistischen Familien aufgenommen, „nicht aber politische Flüchtlinge im Erwachsenenalter, um unerfreuliche Begegnungen zwischen ehemaligen Besatzern und Partisanen in Fabriken und Gaststätten des ,Arbeiter- und Bauernstaats“ auszuschließen“. ${ }^{6}$ Bei den Siegern des griechischen Bürgerkriegs war andererseits die privilegierte Stellung der Bundesrepublik unverkennbar. Athen sei folglich ,der einzige Ort, an dem es die Vertreter Bonns wagten, ehemalige Partisanen als ,Banditen` abzuqualifizieren“; die Verschiebung des Feindbildes lässt sich leicht aus den von Fleischer zitierten Archivalien ablesen, etwa in der Formulierung von 1951, dass „die Erinnerungen an die Untaten der SS [!]“ jetzt in Griechenland etwas verblasst seien dank der „ebenso verwerflichen Untaten der kommunistischen Banditen“, oder, noch eine Steigerung, in der einweisenden These des AA für Theodor Heuss im Rahmen seines Griechenlandbesuches von 1956: „Glücklicherweise sind die Begebenheiten zur Zeit der deutschen Besetzung durch die Grausamkeiten des griechischen Bürgerkriegs überdeckt worden“. ${ }^{7}$

Trotz (aber auch wegen) dieser erinnerungskulturellen Realpolitik des Überdeckens oder Verblassens wurde nach der Stunde Null des Zweiten Weltkriegs der Neuanfang der deutsch-griechischen Beziehungen ermöglicht. Auch vor dem Durchbruch des Anwerbeabkommens zwischen der Bundesrepublik Deutschland und Griechenland am 30. März 1960 und der Einwanderung hunderttausender griechischer Arbeitnehmer:innen, die der wirtschaftlichen Misere jener Begebenheiten und Grausamkeiten entfliehen wollten, konnten in den 1950er Jahren alle deutschen Kulturinstitutionen in Griechenland den Betrieb wieder aufnehmen. 1952 etwa „eröffnet das Goethe-Institut als Nachfolger der Deutschen Akademie weltweit seine erste [...] Auslandsniederlassung in Athen“, ${ }^{8}$ wobei z. B. das Deutsche Archäologische Institut Athen 1951 wieder in Betrieb genommen werden konnte und die Grabungstätigkeiten an den

1963 anführen, die ihre filmische Aufarbeitung in dem Politthriller $Z$ von Costas Gavras fand, und in deren Folge sich mit der Jugendorganisation Lamprakis eine einflussreiche, mehrere Tausend Mitglieder umfassende Bewegung bildete.“ Janis Nalbadidacis: Geburtshelfer der Demokratie. Die Militärdiktatur in Griechenland, 1967-1974, in: Ganzenmüller (Anm. 4), 91-109, hier: 94-95.

6 Hagen Fleischer: Krieg und Nachkrieg. Das schwierige deutsch-griechische Jahrhundert, Köln/Weimar/Wien 2020, 249 und 278.

7 Ebd., 275-276 (Hervorhebung durch Hagen Fleischer).

8 Hagen Fleischer: Europas Rückkehr nach Griechenland. Kulturpolitik der Großmächte in einem Staat der Peripherie, in: Harald Heppner, Olga Katsiardi-Hering (Hrsg.): Die Griechen und Europa. Außen- und Innenansichten im Wandel der Zeit, Köln/Weimar/Wien 1998, 125-191, hier: 169-170. 
traditionellen Stätten sukzessive ihre Fortsetzung fanden. ${ }^{9}$ Vom konservativen Athener Regime wurde dem nicht anerkannten zweiten deutschen Staat diese Behauptung der Kontinuität in der bilateralen Kultur- und Kulturerbepolitik vorenthalten. Lediglich eine begrenzte wirtschaftliche Präsenz wurde zugestanden (z. B. in der Internationalen Messe von Thessaloniki) aber die „Propaganda“ im weitesten Sinne nach Kräften unterbunden. ${ }^{10}$ Obwohl etwa die Brecht-Rezeption schon 1956 ihren Auftakt genommen hat mit einer historischen Aufführung des Kaukasischen Kreidekreises im Athener Kunsttheater des Regisseurs Karolos Koun (1908-1987), übersetzt aus dem Französischen vom späteren Nobelpreisträger Odysseas Elytis (1911-1996), wurde das didaktische Vorspiel mit den beiden Vertretern der kaukasischen Kolchosen ausgelassen, die über das Nutzungsrecht am fruchtbaren Tal und dem besseren Ertrag zum gesellschaftlichen Wohl nach der Vertreibung der deutschen Wehrmacht aus Georgien diskutieren. ${ }^{11}$

Wie lässt sich, in der Perspektivierung solcher Rekontextualisierungen und der schweren Bürde der jüngsten Vergangenheit, die literarische Mobilität, der Literaturtransfer der neugriechischen Literatur in deutscher Sprache im geteilten Berlin vorstellen, im Rahmen jener kulturpolitischen Aktivitäten in Ost und West, mit denen, so der rote Faden der „Berliner Weltliteraturen“, „konkurrierende Communities gestiftet und unterschiedliche Konzepte von Weltliteratur verhandelt“ wurden? ${ }^{12}$ Welche ins-

9 Katja Sporn: Das Deutsche Archäologische Institut Athen - Entwicklung und Bedeutung, in: dies. (Hrsg.) unter Mitarbeit von Themistoklis Bilis: Das Deutsche Archäologische Institut Athen. Architektur und Geschichte, Athen 2018, 8-21, hier: 19.

10 Fleischer (Anm. 8), 171. Vgl. zum deutsch-griechischen Dreieck Dimitrios K. Apostolopoulos: Die griechisch-deutschen Nachkriegsbeziehungen: historische Hypothek und moralischer Kredit. Die bilateralen politischen und ökonomischen Beziehungen unter besonderer Berücksichtigung des Zeitraums 1958-1967, Frankfurt a. M. 2004; Andreas Stergiou: Im Spagat zwischen Solidarität und Realpolitik. Die Beziehungen zwischen der DDR und Griechenland und das Verhältnis der SED

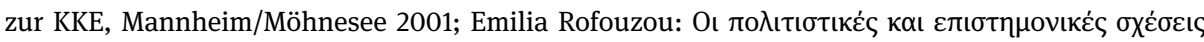

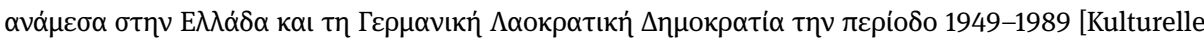
und wissenschaftliche Beziehungen zwischen Griechenland und der Deutschen Demokratischen Republik im Zeitraum 1949-1989], Athen 2010, sowie deren Beiträge: Die griechische Außenpolitik der ersten Nachkriegsjahrzehnte im Licht der Rivalität zwischen DDR und BRD; Das Dreiecksverhältnis BRD-DDR-Griechenland und die „Bereinigung der Kriegsfolgen“ aus der Zeit der Nazi-Okkupation; Einschätzung der auswärtigen Kulturpolitik der DDR im Rahmen ihrer Beziehungen zu Griechenland, in: Hillemann/Pechlivanos (Hrsg.): Deutsch-griechische Beziehungen (Anm. 4), 19-31; 33-44; 113-122.

11 Siehe den Beitrag „Der Brecht der Linken und der Dichtung im Griechenland der 1950er Jahre“ von Zafiris Nikitas im Compendium der deutsch-griechischen Verflechtungen (https://comdeg.eu/compendium/) und vgl. zur Wirksamkeit des Mythos Brecht in Griechenland den Beitrag von Eleni Varopoulou: Das Theater der DDR aus einer griechischen Perspektive, in: Hillemann/Pechlivanos (Hrsg.): Deutsch-griechische Beziehungen (Anm. 4), 147-154.

12 Mein herzlicher Dank gilt dem Literaturarchiv Sulzbach-Rosenberg, insbesondere dem Archivar Michael Peter Hehl, dem Literaturarchiv der Akademie der Künste und der Archivarin Helga Neu- 
titutionalisierten, aber auch nicht gesteuerten Mittler:innen treten auf die Bühne am Fallbespiel der neugriechischen Literatur als Berliner Weltliteratur, und zwar in ihren konflikthaften Positionierungen als vom Staat der Nationalgesinnung propagierte Nationalkultur, als von den politischen Emigranten im Osten im Namen des revolutionären Internationalismus literarisches Exilnetzwerk bzw. als dezentrierte literarische Praxis von Akteur:innen des Austausches?

Und nicht zuletzt: wie verflochten sich die Wege der Vermittlung, da das Bilaterale immer schon multilateral und transnational geprägt ist, wie etwa am Beispiel der neugriechischen Lyrik im Museum der modernen Poesie von Hans Magnus Enzensberger von 1960 zu spüren ist? In dieser „Chrestomathie“ von Enzensberger, dem Lehrbuch einer behaupteten ,poetischen Weltsprache“, da sich Deutschland literarisch seit 1945 in der Lage eines Nachhilfeschülers befände, ${ }^{13}$ ist die neugriechische Lyrik mit Konstantinos Kavafis (1863-1933) und dem künftigen Nobelpreisträger von 1963 Giorgos Seferis (1900-1971) vertreten. Während bei der Übertragung der Gedichte des letzteren durch den Anglisten Christian Enzensberger davon auszugehen ist, dass sich die Wege des Transfers mit der englischen Übertragung der Gedichte von Rex Warner und dem Foyle Prize des Jahres 1961 für Seferis in London kreuzten, trägt die Entdeckung des Alexandriners Kavafis durch Helmut von den Steinen, dem seit 1935 ins Exil nach Griechenland geflüchteten deutsch-jüdischen Georgianer, die unauslösliche Signatur einer Transzendierung des Nationalen und der Nationalliteratur. ${ }^{14}$

\section{Der „Tag der Hellenen“ in der XI. Berlinale (1961): „Weiße Rosen aus Athen“}

Fragt man sich nach dem quasi offiziellen bundesdeutschen Griechenlandbild, das in der „Frontstadt“ Berlin im Jahr des Mauerbaus auch im Namen des Königreichs Griechenland vermittelt wurde, hilft ein Blick in das Programm der XI. Berlinale (23. Juni bis 4. Juli 1961). Es handelt sich nach wie vor um ein bilateral geprägtes Bild,

mann, sowie dem Archiv der BBAW, dem Historischen Archiv des Südwestfunks und dem Allgemeinen Staatsarchiv in Athen, die meine Recherchen mit Archivalien und Kenntnissen unterstützt haben. 13 So Enzensberger an Siegfried Unseld (18. November 1958) und an Peter Suhrkamp (4. März 1959), siehe Meike G. Werner: Vom Annex zum Atelier. Hans Magnus Enzensbergers Museum der modernen Poesie (1960), in: Internationales Archiv für Sozialgeschichte der deutschen Literatur 44 (2019), 399-409, hier: 404-406.

$14 \mathrm{Zu}$ Helmut von den Steinen siehe Chryssoula Kambas: Athen und Ägypten. Helmut von den Steinen, Übersetzer von Kavafis, in: dies., Marilisa Mitsou (Hrsg.): Hellas Verstehen. Deutsch-griechischer Kulturtransfer im 20. Jahrhundert, Köln/Weimar/Wien 2010, 289-328, und vgl. den Beitrag „Zwei Intellektuelle des George-Kreises in Athen der 1930er Jahre“ von Miltos Pechlivanos im Compendium der deutsch-griechischen Verflechtungen (https://comdeg.eu/compendium/). 
das etwa in den Reisebüchern Erhart Kästners (1904-1974), des zeitweiligen Sekretärs von Gerhart Hauptmann und Dolmetschers in Griechenland im Dienste der Wehrmacht, mitgezeichnet wurde. ${ }^{15}$ Am sogenannten „Tag der Hellenen“ der Berlinale wurde das Berliner und internationale Publikum mit der harten Währung Griechenlands auf einen Schlag konfrontiert: mit der griechischen Antike und mit der griechischen Landschaft.

Neben La Notte von Michelangelo Antonioni (Goldener Berliner Bär) und Jean-Luc Godards Une femme est une femme (1. Sonderpreis, Silberner Berliner Bär), die mit ihrer Kälte die am 13. August 1961 vom Kalten Krieg zu schaffenden brutalen Fakten quasi vorausahnten (so die Berlinale-Chronik), ${ }^{16}$ waren im Wettbewerb zwei griechenlandbezogene Filme zu sehen. Einerseits als offizieller Beitrag Griechenlands die sich eng an die antike Vorlage von Sophokles haltende Verfilmung der Antigone von Giorgos Tzavellas (1916-1976), mit der Schauspielerin Irene Papas ( ${ }^{\star 1926)}$ in der Titelrolle, andererseits, unter den fünf von Alfred Bauer zum Wettbewerb eingeladenen Filmen, der bundesdeutsche Dokumentarfilm Wolfgang Mueller-Sehns Traumland der Sehnsucht, der den Sonderpreis (Silberner Berliner Bär) in dieser Kategorie gewann. ${ }^{17}$

Als langer Dokumentarfilm aus deutscher Touristik-Perspektive wurde Traumland der Sehnsucht für die idyllischen Panorama- und Landschaftaufnahmen gepriesen; die Berliner Morgenpost etwa urteilte am 29. Juni zum „Tag der Hellenen“:

\begin{abstract}
Wolfgang Müller-Sehn und seine Frau Lilo haben es sich nicht leicht gemacht. Zwei Jahre lang sind sie mit Auto, Schiff und Flugzeug kreuz und quer durch Griechenland gereist, haben einsame Klöster, antike Tempel, lärmende Märkte, elegante Straßen und stille Dörfer besucht, haben arbeitende und feiernde Menschen und sonnenüberflutete Landschaften mit der Farbkamera festgehalten. Das Ergebnis ist nicht etwa ein wahlloses Kunterbunt an Eindrücken geworden, sondern eine in aller Vielfalt harmonisch geordnete Verbindung von Antike und Gegenwart, von Glanz und Armut, Schönheit und Leben. Man wird verzaubert von einer Fremde, die man nicht als fremd empfindet. ${ }^{18}$
\end{abstract}

Diese Mythisierung von Geschichte und Volkscharakter sticht schon mit den ersten Sätzen des Filmes ins Auge:

$15 \mathrm{Zu}$ Erhart Kästner und Griechenland siehe Helga Karrenbrock: Erhart Kästners Griechenland, in: Chryssoula Kambas, Marilisa Mitsou (Hrsg.): Die Okkupation Griechenlands im zweiten Weltkrieg. Griechische und deutsche Erinnerungskultur, Köln/Weimar/Wien 2015, 391-398, und Nafsika Mylona: Delphi und der Mythos des Nationalsozialismus. Politisch-religiöse Implikate in Franz Spundas und Erhart Kästners Ortsbeschreibungen, in: ebd., 399-408.

16 Online abgerufen am 10. Mai 2021 unter https://www.berlinale.de/de/archiv/jahresarchive/1961/01_jahresblatt_1961/01_jahresblatt_1961.html.

17 Wolfgang Jacobsen: 50 Jahre Berlinale. Internationale Filmfestspiele Berlin, Berlin 2000, 99 und 106. 18 „Der Tag der Hellenen“, in: Berliner Morgenpost (29.06.1961), zitiert nach http://www.weisse-rosen-aus-athen.de/1961.htm, online abgerufen am 10. Mai 2021. 
Griechenland, Sehnsucht der Jahrhunderte, Ziel aller, die träumen vom sonnigen Süden, vom blauen Meer und marmornen Göttersteinen. Auch uns zog diese Sehnsucht nach dem Land der Griechen, wo Europas Herz zu schlagen und ein Geist zu fragen begann. Denn das alte Hellas ist nicht tot. Es lebt weiter in immer neuen Verwandlungen. Es ist ein Land geballt durch die Größe seiner Vergangenheit, geladen mit Armut, und doch immer wieder durchklungen von der Freude des Lebens, die sich durch keine Not unterkriegen lässt. ${ }^{19}$

Die Dreharbeiten, aus denen auch zwei 1964 erschienene Fotobände von Lilo MuellerSehn in der Reihe „Europas Ferienstrassen“ hervorgingen, ${ }^{20}$ wurden auf Anfrage der Botschaft der Bundesrepublik Deutschland in Athen vom griechischen Königshaus unterstützt. „M. Müller-Sehn m’été recommandé par mon Gouvernement, et je suis plein de confiance qu'il réussira à créer un film de première qualité digne d'être projeté dans le monde entier“, lautete am 13. Oktober 1959 die Empfehlung, damit im Palais Royal gedreht werden durfte. ${ }^{21}$ Die filmische Verzauberung brachte es ja auf den Punkt: der König gehöre zu Hellas wie Hellas zu seinem König. ${ }^{22}$

Auch wenn das Königspaar bei der Berliner Premiere nicht anwesend war, nahmen an der österreichischen in Salzburg Königin Friederike (ursprünglich Prinzessin von Hannover und Herzogin zu Braunschweig-Lüneburg) sowie ihre Tochter, Prinzessin Sophia, und deren Verlobter, Prinz Juan Carlos, teil, wobei der Regisseur Wolfgang Mueller-Sehn, Lilo Mueller-Sehn sowie der Generaldirektor und der Publicity Manager

19 Der Film ist unter https://www.youtube.com/watch?v=0Oqt35CgG3o\&feature=share zu sehen (online abgerufen am 10. Mai 2021); für das Zitat siehe 0:20-0:58. Im Vorspann des Filmes wird unter beratender Funktion („Kommentar-Mitarbeit“) Johannes Gaitanides (1909-1988) erwähnt, der mit seinem Bestseller Griechenland ohne Säulen das nationalsozialistische und später das bundesrepublikanische Griechenlandbild der Adenauer-Ära stark mitgeprägt hat; siehe dazu Miltos Pechlivanos: Zum historischen Gedächtnis der Geisteswissenschaften. Die deutsche Neogräzistik und die Okkupation Griechenlands, in: Kambas/Mitsou (Hrsg.): Die Okkupation Griechenlands im zweiten Weltkrieg (Anm. 15), 353-372, und vgl. den Beitrag „Die Geburt des Griechenlandbildes in der Bundesrepublik aus dem Geiste des Nationalsozialismus: Die Griechenland-Bücher von Erhart Kästner und Johannes Gaitanides im Spannungsfeld zwischen Erneuerung und Kontinuität“ von Athanasios Anastasiadis im Compendium der deutsch-griechischen Verflechtungen (https://comdeg.eu/compendium/).

20 Lilo Mueller-Sehn: Griechische Reise. Von Saloniki bis zur Südspitze des Peloponnes, Wien/München 1964, und dies., Griechische Inseln. Von Korfu bis Rhodos - Von Lesbos bis Kreta, Wien/München 1964. Den Tenor verrät schon die im Vorwort des ersten Fotobandes gestellte Frage: „Wo hört der Mythos auf, wo fängt die Geschichte an? Man weiss es nicht.“

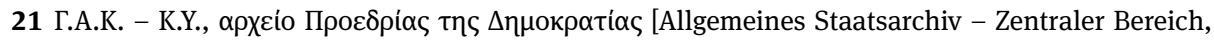
Archiv der Präsidentschaft der Republik], Signatur f. 144.

22 Im Film (41:20-42:00) lautet der Text: „König Paul und Königin Friederike haben die schwere Aufgabe, ihr Land aus den Nachwehen zweier Kriege und eines blutigen Bürgerkrieges herauszuführen. Dies Land, das durch schwere politische Hypotheken belastet ist, durch wirtschaftliche Probleme ohne Ende, ganz zu schweigen von den innerpolitischen Spannungen, die nicht zuletzt aus der krassen Eigenwilligkeit des Griechen hervorgehen. Die Königsfamilie hat mit Überlegenheit und Charme, mit fürsorgender Liebe und politischem Geschick manche Schwierigkeiten ausgleichen können. Das Volk liebt und verehrt sie. Der König gehört zu Hellas wie Hellas zu seinem König.“ 
der Columbia-Filmgesellschaft mit griechischen Orden ausgezeichnet wurden. ${ }^{23}$ Den Film erwähnt Königin Friederike auch in ihrer Autobiographie auf den dem Staatsbesuch des griechischen Königspaares in der Bundesrepublik im September 1956 gewidmeten Seiten:

\begin{abstract}
Wherever we went there were friendly laughing faces. It was as if people wanted to say, forget what happened during the war. We love Greece and the Greek people. The invasion of Greece during the war had been very unpopular with the German public. The German intellectual world is strongly Greek orientated and knowledge and love of our classics have made Greece a true „dreamland of their longing“, I quote the name of a German cultural film on Greece. We left, greatly pleased to have re-established happy personal relations with a land traditionally pro-Greek, a land in which I was born, and which, through a freak of history, had brought so much suffering to Greece, the country of my love. ${ }^{24}$
\end{abstract}

Wie es sich auch verhalten mag mit einer so apostrophierten „Laune der Geschichte“, der Dokumentarfilm von Mueller-Sehn erhielt neben dem Sonderpreis der Berlinale die Kulturfilmprämie 1961 des Bundesinnenministeriums, auch wenn bei der Filmbewertungsstelle Wiesbaden eine Meinungsverschiedenheit zu Tage kam: hatte sich der Bewertungsausschuss für das Prädikat „Wertvoll“ entschieden, da der Film alle sozialen und politischen Probleme umginge, vor allem die moderne Arbeitswelt in Griechenland, „um sich ganz auf die stilleren Bezirke des seit der Antike fast unveränderten Lebens an den Küsten und auf den Inseln zu konzentrieren“, erteilte der Hauptausschuss das höchste Prädikat „Besonders Wertvoll““25

Seine langwierige Wirkung als Verflechtung auswärtiger Kulturpolitik, Propaganda und Tourismus-Marketing verdankt das Traumland der Sehnsucht jedoch der Musik von Manos Hadjidakis (1925-1994), der 1960 mit „Ein Schiff wird kommen“ für den Film „Sonntags ...nie“ den Oscar für das beste Lied gewonnen hatte, und der

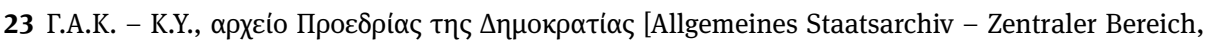
Archiv der Präsidentschaft der Republik], Signatur f. 202.

24 Frederica Queen of the Hellenes: A Measure of Understanding, London 1971, 202. Zum Staatsbesuch des griechischen Königspaares vgl. Apostolopoulos (Anm. 10), 65-68.

25 Online abgerufen am 10. Mai 2021 über die Datenbank Dokumentarfilmgeschichte, unter http:// www.db.dokumentarfilmgeschichte.de/detail.php?typ=film\&id=13294. Die ostdeutschen Pendants zum „Traumland der Sehnsucht“ dürfen hier nicht unerwähnt bleiben, die fünf Kurzfilme („Hellas ohne Götter“, „Insel der Rosen“, „Bilder aus Athen und Rhodos“, „Zwischen Himmel und Erde“ und „An der Via Egnatia“), die Karl Gass, Künstlerischer Leiter des DEFA-Studios für Populärwissenschaftliche Filme, in und über Griechenland 1957/58 gedreht hat, vgl. Ines Walk: Art. „Karl Gass“, in: Biographien von KünstlerInnen der DEFA, online abgerufen am 10. Mai 2021 auf der Homepage der DEFA Stiftung unter https://www.defa-stiftung.de/defa/kuenstlerinnen/kuenstlerin/karl-gass/. Im Rahmen der deutsch-deutschen Konkurrenz sollten in diesen Filmen - so Giorgos Vrazitoulis, der die Vorführung der Filme im Rahmen der Konferenz Deutsch-griechische Beziehungen im ostdeutschen Staatssozialismus (1949-1989) des Centrum Modernes Griechenland der Freien Universität Berlin (22.23.04.2015) organisiert hatte - „nicht nur die antiken Stätten und sonstige touristische Attraktionen, sondern insbesondere die Armut, die sozialen Gegensätze in Griechenland sowie seine politische Abhängigkeit von den USA in den Vordergrund treten“. 
Stimme von Nana Mouskouri ( ${ }^{\star}$ 1934), die mit dem „Traumland der Sehnsucht“ ihre internationale Chance bekommen hatte und am 4. Juli ihre griechischen Chansons zwischen Volksmusik und westlichen Schlagern in einem Berliner Ton-Studio auch in deutscher Sprache auf Schallplatten aufnehmen wird. ${ }^{26}$

Während es sich aber bei der griechischen Version der Melodie der „Weißen Rosen aus Athen“ um die Vertonung eines Gedichtes des Lyrikers des neugriechischen Modernismus Nikos Gatsos (1911-1992) mit dem Titel „Wenn du drei Mal pfeifst“ handelte, eines Lyrikers, der Songtexte für mehrere Projekte von Hadjidakis geschrieben hatte, trug die touristische Rekontextualisierung in deutscher Sprache mit dem neuen Text der Sehnsucht nach Urlaub, nach Sonne und nach Meer, und dem Fernweh des Nachkriegseskapismus der deutschen Schlager Rechnung. Die Autobiographie der Sängerin und später auch Europa-Abgeordnete für die Konservativen Nana Mouskouri trägt nach wie vor den Titel Stimme der Sehnsucht.

\section{Ostberlin: Johannes Irmscher, Melpo Axioti und Dimitris Hadzis}

Es waren nicht die 1128 Jugendlichen aus kommunistischen Familien, 1949/50 bei der Aufteilung der Exilierten nach dem Bürgerkrieg in der damaligen Sowjetischen Besatzungszone und späteren DDR aufgenommen und in Radebeul bei Dresden untergebracht, die Ostberlin zu einem Zentrum der neugriechischen Exilliteratur während des Kalten Krieges machten. Die Ausnahme, die die Regel bestätigt, ist der Schriftsteller, Übersetzer und Kulturmittler Thomas Nicolaou (1937-2008), der mit seinem deutschsprachigen Debüt-Roman Nachts kamen die Barbaren (1968) einen historischen Bogen zwischen dem griechischen Bürgerkrieg und der Obristendiktatur spannte, in literatursoziologisch überaus interessanten Milieus stand ${ }^{27}$ und, wie wir seit 1992 wissen, als Mitarbeiter der Staatssicherheit mit dem Namen „Anton“ nicht nur seine griechischen Landsleute, sondern auch zahlreiche Künstler:innen und Schriftsteller:innen der DDR, u. a. Stefan Heym, Rainer Kunze, Volker Braun und Wolf Biermann, wie auch das befreundete Ehepaar Gerhard und Christa Wolf bespitzelt hatte. ${ }^{28}$ Letztere, die in ihrer Erzählung Sommerstück (1989) die gemeinsamen Sommer

26 Der Tagesspiegel (28.06.1961), zitiert nach zitiert nach http://www.weisse-rosen-aus-athen.de/ soundtrack.htm, online abgerufen am 10. Mai 2021.

27 Denis Püllmann: Thomas Nicolaous Roman Nachts kamen die Barbaren im literarischen Feld der DDR, in: Hillemann/Pechlivanos (Hrsg.): Deutsch-griechische Beziehungen (Anm. 4), 135-145, nennt die Künstlerkolonie Drispeth oder das Chemische Kombinat Buna (hier: 135).

28 Siehe Stratos N. Dordanas, Vaios Kalogrias: Die Stasi und ihre Griechen, in: Hillemann/Pechlivanos (Hrsg.): Deutsch-griechische Beziehungen (Anm. 4), 93-109, hier: 107-108, und vgl. ders.: Oı

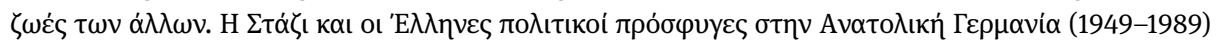


im mecklenburgischen Drispeth verarbeitet hat, begleitete Nicolaou 1982 auf der Griechenland-Reise, die den Anstoß zu der Erzählung Kassandra gab, welche wiederum 1983 von Nicolaou ins Griechische übersetzt wurde. ${ }^{29}$

Es war das Verdienst von Johannes Irmscher (1920-2000), führendem Fachvertreter der Klassischen Philologie, der Patristik und der Byzantinistik in der DDR und seit 1966 jahrelangem Vizevorsitzenden des „Komitees DDR-Griechenland“, ${ }^{30}$ die Chance in den späten 1950er Jahren ergriffen zu haben, die Neogräzistik an der Deutschen Akademie der Wissenschaften zu Berlin zu begründen und, vor dem Hintergrund der transnationalen Funktion des sowjetischen Weltliteraturprojekts für die anderen Staaten des Ostblocks, den Literaturtransfer zu intensivieren. Der wissenschaftspolitische Vernetzungswille Irmschers ging Hand in Hand mit der externalisierenden Gedächtnispolitik der DDR, mit der die fortwährende Schuld für den Zweiten Weltkrieg systembedingt der Bundesrepublik überlassen wurde. Die enge Zusammenarbeit Irmschers mit den griechischen Kommunisten in der Emigration hatte einen ersten Höhepunkt in der 1957 in Berlin veranstalteten Konferenz „Probleme der neugriechischen Literatur“, deren Protokoll in vier Bänden der „Berliner Byzantinischen Arbeiten“ 1959 und 1960 beim Akademie Verlag vorgelegt wurde. Auf die Frage des Vertreters der Kammer für Außenhandel der DDR in Griechenland nach den Zielen einer Konferenz, die in Zeiten des Kalten Krieges das Lager der Forscher:innen von Ost und West zusammenzuführen beanspruchte, hat Irmscher am 29. Januar 1957 folgendermaßen geantwortet:

Die Konferenz ist meines Wissens die erste Tagung, welche außerhalb Griechenlands über Fragen der neugriechischen Literatur abgehalten wird. Ich glaube, diese Tatsache ist ein nicht zu unterschätzendes Politikum und wird ohne Zweifel in Griechenland gewürdigt werden (wobei man auch vielleicht nicht übersehen wird, dass es gerade ein sozialistischer Staat ist, der diese Initiative ergreift). Durch die Konferenz wird weiter die Stellung unserer Akademie als eine Stätte des wissenschaftlichen Austausches zwischen Ost und West gefestigt werden; da zu erwarten ist, dass auch die ersten Vertreter des Fachgebietes zu unserer Konferenz kommen werden, oder sie zumindest ihr tätiges Interesse erklärt haben, besteht zu hoffen, dass die Zusammenkunft Gelegenheit geben wird, wissenschaftliche Verbindungen zu erneuern oder neue anzuknüpfen. Speziell für Griechenland erhoffe ich von der Konferenz eine persönlich Fühlungnahme mit den führenden Fachvertretern - was für Saloniki bereits als gesichert gelten kann -, von daher eine ständige Zusammenarbeit, wie sie zwischen Berlin und Athen in den zwanziger Jahren bereits sehr rege war, und zwar eine solche wissenschaftliche Zusammenarbeit, die zugleich eine Verstärkung des Einflusses unseres Staates in Griechenland bedeutet (den man ja dort wegen des vielen Reiseverkehrs weithin überhaupt nicht kennt). ${ }^{31}$

[Die Leben der Anderen. Stasi und die griechischen politischen Emigranten in Ostdeutschland (19491989)], Thessaloniki 2020, 198-209.

29 Siehe den Artikel „Thomas Nicolaou“ von Marco Hillemann im Compendium der deutsch-griechischen Verflechtungen (https://comdeg.eu/compendium/).

30 Ich folge hier meinen Ausführungen in Pechlivanos (Anm. 19), 354-362.

31 Berlin-Brandenburgische Akademie der Wissenschaften, Akademiearchiv, Signatur ZIAGA, A 3940/1. 
Auch wenn mehr als 350 Wissenschaftler:innen und Schriftsteller:innen in Europa und in den Staaten zwischen 1956 und 1957 den Aufruf mit der Unterschrift des Präsidenten der Akademie Max Volmer erhielten - unter diesen einige der wichtigsten Vertreter:innen der Literatur in Griechenland ${ }^{32}$ - zur Konferenz versammelten sich nur die wichtigsten Vertreter:innen der griechischen linken Intelligenz im Ostblock, Autorinnen wie Elli Alexiou (1894-1988), Foula Hadzidaki (1906-1984), und Melpo Axioti (1903-1973), und Autoren wie Thodosis Pieridis (1908-1968) und Dimitris Hadzis (1913-1981) bzw. Stratis Tsirkas (1911-1980) aus der griechischen Diaspora in Ägypten. Die Entscheidung der griechischen Regierung, den eingeladenen griechischen Autor:innen oder Wissenschaftler:innen kein Visum zu erteilen, übertraf alle pessimistischen Erwartungen und zeigte, dass wissenschaftlicher Austausch gegenseitigen Willen braucht. Die einzige Stimme Griechenlands, die im Frühjahr 1957 in Ostberlin zu hören war, war somit auf Tonband, die Stimme des Architekten und Volkskundlers Kostas Biris (1899-1980). ${ }^{33}$

Diese unzeitgemäße (und $\mathrm{zu}$ ambitionierte) Initiative bot jedoch eine wichtige (wenn auch wiederum verpasste) Gelegenheit für die Positionierung der neugriechischen Literatur im Netzwerk der (Ost)Berliner Weltliteraturen. Zwei der bedeutendsten griechischen Autor:innen des 20. Jahrhunderts, Melpo Axioti und Dimitris Hadzis, hatten sich in Ostberlin niedergelassen, Axioti als Gastlektorin ab dem Wintersemester 1958/59 bis 1964 am Institut für Altertumswissenschaften der Humboldt Universität und Hadzis zwischen 1957 und 1963 als wissenschaftlicher Mitarbeiter und Doktorand von Irmscher am Institut für Altertumskunde der Deutschen Akademie der Wissenschaften zu Berlin.

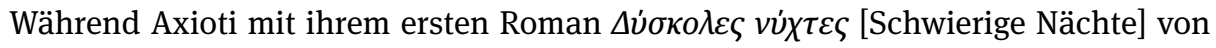
1938 sich dem inneren Monolog der literarischen Moderne zuwandte (,ich schrieb [...] ohne einen theoretischen Ansatz, nur mit dem Herzen, nach meinem eigenen Geschmack und meinem Instinkt“, notierte sie später), wurde die Literatur für die Widerstandskämpferin während der deutschen Besatzung „Waffe und Werkzeug“ zur Unterstützung des Kampfes; die grande dame der griechischen engagierten Literatur,

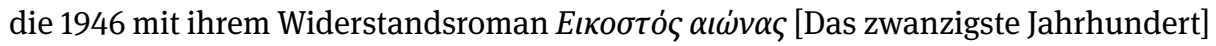
in Übersetzungen ins Französische und Deutsche - 1949 unter dem Titel Tränen und

32 Unter anderen waren etwa Nikos Kazantzakis (1883-1957), Galateia Kazantzaki (1881-1962), Tasos Livaditis (1922-1988), Stratis Myrivilis (1890-1969), Lilika Nakou (1904-1989), Kosmas Politis (18881974), Jannis Ritsos (1909-1990), Vassilis Rotas (1889-1977), Kostas Varnalis (1884-1974), Ilias Venesis (1904-1973) und Nikiforos Vrettakos (1912-1991) eingeladen.

33 Johannes Irmscher: Die Konferenz über Fragen der neugriechischen Literatur in Berlin April 1957,

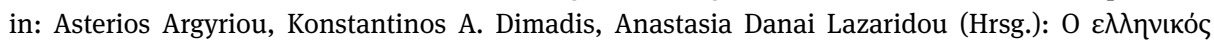

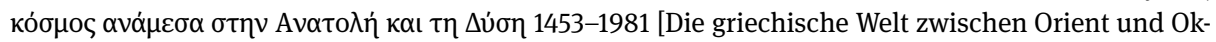
zident 1453-1981], Athen 1999, 285-292, hier: 288 f.: „möglicherweise suchte man auch zu verhindern, dass griechische Intellektuelle, die als politische Flüchtlinge im Ausland lebten, mit ihren Landsleuten in persönlichen Kontakt traten.“ 
Marmor, mit einem Vorwort von Anna Seghers - und dann auf Italienisch, Russisch, Bulgarisch und Polnisch den internationalen Durchbruch erlebte, floh 1947 nach Paris, wurde dort 1949 ehrenvoll in den Vorstand des 1. Weltkongresses des Weltfriedenrates berufen, aber im September 1950 ausgewiesen und in die DDR abgeschoben, wo sie sich schließlich in den Jahren 1957-1964 niederließ, nach einer Zeit des Umherirrens zwischen 1952 und 1957 in Warschau und kurzfristig in Sofia. ${ }^{34}$

Mit der Ausnahme weniger deutscher Schriftsteller:innen mit Französischkenntnissen, etwa Paul Wiens, der aus dem Französischen 1961 ihre Dichtung Konterbande ins Deutsche übertragen hat, bzw. dem Übersetzer von Tränen und Marmor Kurt Stern, ${ }^{35}$ blieb das Leben in Berlin für Axioti bloß „Transit und Provisorium“ im Hotel Johannishof, Gästehaus des DDR-Ministerrates, wo sie auch von ihren Student:innen zum Unterricht besucht wurde. ${ }^{36}$ Es war die Zeit der Entstehung eines der wichtigsten

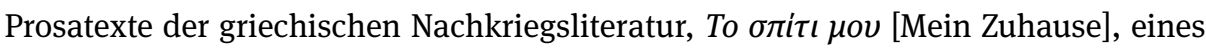
metafiktionalen Textes, mit dem Axioti zum Modernismus der Zwischenkriegszeit zurückkehrt; auf der letzten Seite des Buches, das direkt nach ihrer Repatriierung 1965 in Athen veröffentlicht wurde, schreibt sie über sich in dritter Person vom Zustand des Exils: „Es ist ein Glücksfall, dass sie während ihrer Abwesenheit alle Wörter ihrer Sprache bewahrte und sicher noch die Namen aller Winde kennt. “37

Der jüngere Hadzis, seit 1935 und bis 1952 Mitglied der KP, zweimal 1936 und 1947 auf den Internierungsinseln, nach dem Bürgerkrieg im Exil in Bukarest, Budapest und Ostberlin bis zu seiner Repatriierung in Griechenland nach der Militärdiktatur, wo er 1952 in Abwesenheit zum Tode verurteilt worden war - sein Bruder wurde 1948 exekutiert -, war als Journalist tätig, bis er den Versuch unternahm, so schrieb er in einem Brief an seine Schwester Litsa in Griechenland am 26.06.1957, als Neogräzist in Budapest und in Ostberlin „einen Teil der verlorenen Zeit wiederzugewinnen““. ${ }^{38}$ Für

34 Vgl. Maria Kakavoulia: Melpo Axioti (1903-1973), Leben und Werk, in: Melpo Axioti: Kadmo, übers. von Maria Zafón, Berlin 2017, 79-113; die Zitate hier: 82. Vgl. Dennis Püllmann: Melpo Axioti und Dimitris Chatzis. Zwei griechische Schriftsteller im Ostberliner Exil, in: Margrid Bircken, Andreas Degen (Hrsg.): Reizland DDR. Deutungen und Selbstdeutungen literarischer West-Ost-Migration, Göt-

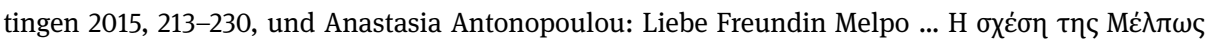

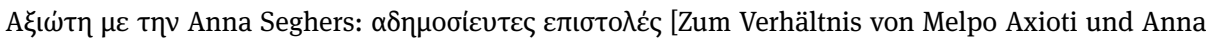

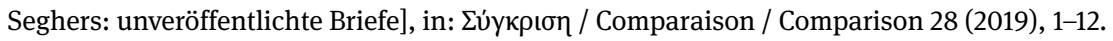

35 Zur Freundschaft Axiotis mit Kurt und Jeanne Stern siehe Antonopoulou, ebd., sowie Anm. 13 und 31. 36 Püllmann, ebd., 223.

37 Zitiert nach Kakavoulia (Anm. 34), 92.

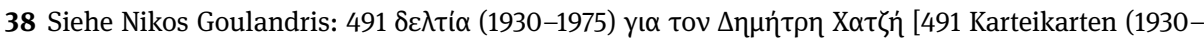
1975) zu Dimitris Hadzis], Athen 2001, 170. Dimitris Hadzis und seinem Beitrag zur neugriechischen Kulturgeschichte war der erste Workshop der Reihe „Konstruktionen der neugriechischen Kultur“ (19.-20.12.2008) im Institut für Griechische und Lateinische Philologie an der Freien Universität Berlin gewidmet; online abgerufen am 10. Mai 2021 auf der Webseite der Freien Universität Berlin, unter https://www.geisteswissenschaften.fu-berlin.de/we02/institut/termine/termine_archiv/ngr_veranstaltung_hadzis.html. 
die Berliner Zeit ist seine Akte im Akademiearchiv der BBAW aufklärend, als etwa im November 1958 die Abteilung Außenpolitik und Internationale Verbindungen der SED von der Abteilung Kader und Arbeit erfahren wollte, wie es um die Parteimitgliedschaft und um die fachliche und politische Betätigung des politisch Verfolgten stehe, aber auch wieso „der an der Akademie tätige Grieche Chatzis in Westberlin gesehen wurde". ${ }^{39}$

Obwohl Hadzis auf Befragung hin angab, ,am Bahnhof Zoo gewesen zu sein, um dort seine für 3 Tage aus Athen nach Berlin kommende Schwester vom Bahnhof abzuholen“ und erklärte, „dass er sonst nie nach Westberlin ginge“, spricht die Antwort der Leiterin an das Zentralkomitee Bände: Hadzis sei allgemein als intellektueller Einzelgänger bekannt, mit einer pazifistischen Grundeinstellung und unklarer Einstellung zur volksdemokratischen Ordnung; er halte sich möglichst fern vom gesellschaftlichen Leben der Akademie und von der Gemeinschaft der griechischen Emigranten in Berlin und lege dem politischen Geschehen gegenüber ein ungewöhnliches Desinteresse an den Tag. Auch wenn die Berichterstatterin nicht klarstellen konnte, warum er selbst kein Mitglied der KPG sei, ,da die Verständigung mit ihm in deutsch äußerst schwierig ist“, mutmaßte Irmscher, die Gründe „mögen in den mannigfachen Auseinandersetzungen zu suchen sein, welche innerhalb der Kommunistischen Partei Griechenlands nach der militärischen Niederlage geführt wurden“. ${ }^{40}$

Wäre man damals in der Lage gewesen, die titelgebende Erzählung von Hadzis’

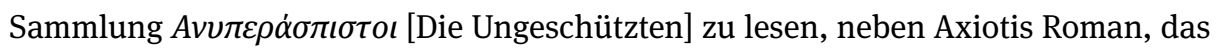
zweite der bedeutendsten Werke der erzählenden griechischen Nachkriegsliteratur, die in der DDR entstanden, hätte man vielleicht einen trefflichen Grund. Es handelt sich in dieser Sammlung um den ersten literarischen Versuch ,(nicht nur der politischen Flüchtlinge, sondern der gesamten Literatur über den Bürgerkrieg), das Trauma als ein geteiltes darzustellen, als ein Trauma, das die beiden kriegführenden Lager gleichermaßen betrifft“; ${ }^{41}$ im Exil sei somit eine „Anerkennung des Traumas des Anderen“42 vollzogen worden.

Während ihrer Berliner Jahre waren Melpo Axioti und Dimitris Hadzis sehr engagiert in der Vermittlung der neugriechischen Literatur in deutscher Sprache gewesen. Behauptete Irmscher noch 1957, dass die nach 1945 in der DDR erschienenen Bücher der politischen Emigranten daran kranken, „dass sie nicht direkt aus dem

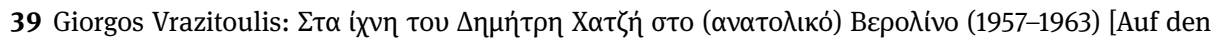
Spuren von Dimitris Hadzis in (Ost)Berlin (1957-1963)], in: Exantas. Eine griechisch-deutsche Kulturzeitschrift 11 (Dezember 2009), 62-75, hier: 69-70.

40 Ebd. - Für die Planung eines Wiedersehens der Geschwister nach zwanzig Jahren vgl. Goulandris (Anm. 38), 164-165.

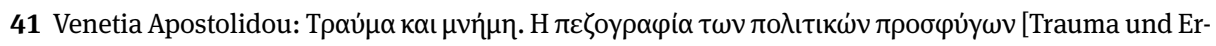
innerung. Die Prosa der politischen Emigranten], Athen 2010, 24, zitiert nach Püllmann (Anm. 34), 228. 42 Ebd. 
Griechischen vorgenommen wurden, sondern sich auf die französischen Ausgaben stützten“, 43 ermöglichte die neue Konstellation um 1960 eine Rezeption der neugriechischen Literatur unter dem doppelten Vorzeichen des sowjetisch inspirierten Ostberliner Weltliteraturprojektes und der externalisierenden Gedächtnispolitik der DDR.

Mit der 1960 beim Verlag Volk und Welt erschienenen Anthologie neugriechischer Erzählungen Antigone lebt, dessen Titel die klassische Antike und den antifaschistischen Widerstand zusammenknüpft, ${ }^{44}$ haben Axioti und Hadzis u. a. etliche Autor:innen ausgewählt, die sich mit der Thematik der deutsch-griechischen Verflechtungsgeschichte auseinandersetzten und eine literaturgeschichtliche sowie gattungshistorische Einführung geliefert. Den Spuren der offiziellen DDR-Rezeption lässt sich im Bundesarchiv und im Archiv der Akademie der Künste nachgehen, bei den Druckgenehmigungsvorgängen zu Verlagspublikationen, sowohl für Antigone lebt als auch für die weiteren ins Deutsche übersetzten und in den frühen 1960er Jahren veröffentlichten Werke griechischer Nachkriegsliteratur, z. B. die Romane von Menelaos Ludemis Ein Kind zählt die Sterne (1960), von Andreas Frangias Menschen und Häuser (1961) oder der im Exil lebenden Autorin Elli Alexiou Die dritte christliche Mädchenschule (1963) bzw. die Erzählungen Das zerstörte Idyll (1965) von Dimitris Hadzis und die autobiographische Schilderung des Athener Geisellagers Haidari im Leben auf Widerruf (1964) von Themos Kornaros (1906-1970)..$^{45}$

Die bundesrepublikanische Öffentlichkeit wurde erst in der zweiten Hälfte der 1960er Jahre auf die im Osten übersetzte und herausgegebene neugriechische Literatur aufmerksam. Knapp drei Monate nach dem Militärputsch vom 21. April Juli 1967 konnten die Zeit-Leser:innen den Beitrag des Germanisten Helmut Salzinger lesen:

43 Siehe oben, Anm. 31.

44 Der Titel der Anthologie nimmt den Anlass von der Erzählung „Antigoni“ von Ilias Venesis, in der in Anlehnung an den antiken Stoff geschildert wird, wie trotz des Verbots eine Schwester ihren von deutschen Soldaten erhängten Bruder begräbt.

45 Aus dem Kreis dieser Autor:innen wurden auch um 1960 die offiziellen Einladungen für Reisen in die DDR vom Deutschen Schriftstellerverband ausgesprochen. 1958 wurde Ludemis nach Ostberlin eingeladen und 1961 Kornaros. 1962 kam Ritsos nach Ostberlin, dessen deutschsprachige Rezeption sich vor 1967 auf Stephan Hermlins Übersetzung eines Auszuges aus „Brief an Joliot Curie“ (1951) in der Berliner Zeitschrift Aufbau und auf die 1957 und 1960 in Sinn und Form erschienenen „Die Mondscheinsonate“ und „Das Fenster“ (siehe unten Anm. 65, 137) beschränkte, und 1966 kam Iakovos Kambanellis (1930-2010), dessen Chronik Mauthausen, ein Bericht seiner Erlebnisse im Konzentrationslager, 1961 in Athen erschien und dessen Mauthausenlieder in der Vertonung von Mikis Theodorakis 1966 uraufgeführt wurden, gesungen von Maria Farantouri, die somit ihren internationalen Durchbruch schaffte. - Mein herzlicher Dank gilt Jutta Müller-Tamm, die mir die im Rahmen des Projekts „Writing Berlin“ gesammelten Daten der einreisenden Schriftsteller:innen in die DDR zur Verfügung gestellt hat. 
Die große Zahl der politisch engagierten und zum Sozialismus tendierenden Dichter Griechenlands wird dem literarischen Bewusstsein der Bundesrepublik vorenthalten. Ob das bloßer Zufall ist? Wer sich über diesen Teil der neugriechischen Literatur orientieren will, sieht sich - es muss leider gesagt werden - auf die Buchproduktion der DDR verwiesen, obschon auch dort nicht gerade viel übersetzt wurde. Immerhin erschien dort die Prosaanthologie Antigone lebt, in der viele der bei uns verheimlichten Autoren vertreten sind, wenn auch nicht immer mit ihren besten Werken. Weiterhin erschienen in der DDR einige Romane, die es durch ihre Darstellung des griechischen Alltags begreiflich machen, warum so viele Griechen ihre Hoffnungen auf den Sozialismus setzen. [...] Gewiss, die ungeschminkte Darstellung der sozialen Verhältnisse des Landes verrät diese Autoren sämtlich als Sozialisten oder Kommunisten, und damit schon könnte einem Bundesrepublikaner ihre Verfolgung und Unterdrückung gerechtfertigt erscheinen. Als ob es keine Menschen wären, die sich da gegen die himmelschreienden Zustände im wirtschaftlich und sozial rückständigsten Land Europas empören. Diese Empörung, die beinahe schon eine Sache des persönlichen Anstandes ist, braucht nicht immer mit einer parteipolitischen Bindung gekoppelt zu sein. ${ }^{46}$

\section{Westberlin: Das Berliner Künstlerprogramm und die Mittler:innen}

In dieser Konstellation des griechischen „dreißigjährigen Krieges“ im Inneren, der mit der Obristendiktatur die letzten sieben Jahren abschloss, und der neugriechischen engagierten (Exil-)Literatur als einer in den Debatten des Realismus, Sozrealismus oder kritischen Realismus wie im sowjetisch inspirierten Ostberliner Weltliteraturprojekt verstrickten Literatur, welcher konnte der Ort Griechenlands in der Westberliner Kulturoffensive der frühen 1960er Jahre sein? Geht man davon aus, dass erst ab 1967 die jüngere Generation um Mikis Theodorakis und die Jungendbewegung der 1960er Jahre, wie etwa Vassilis Vassilikos (`1934) mit seinem Roman Z, die internationale Bühne als von der Junta Vertriebene betreten würde, bliebe dann noch die Vorreiterfunktion jener griechischen Diaspora, die nach dem Zweiten Weltkrieg das Land verlassen und sich für eine der westeuropäischen Metropolen entschieden hatte bzw. entscheiden musste.

Um vom älteren Nikos Kazantzakis ganz zu schweigen, der das letzte produktive Jahrzehnt seines Lebens im Südosten Frankreichs, in Antibes, verbracht hatte und in der Universitätsklinik Freiburg gestorben war, soll hier eine Initiative des Athener Französischen Institutes und dessen Direktoren Octave Merlier und Roger Milliex nicht unerwähnt bleiben. Ende 1945 schickten sie mit einer außergewöhnlich hohen Anzahl von Stipendien mehr als 150 Wissenschaftler:innen und Künstler:innen aus Griechenland nach Frankreich, damit diese ihre Studien fernab des schädlichen Klimas des

46 Helmut Salzinger: „Die Austreibung des Geistes. Zur Verfolgung der Schriftsteller in Griechenland“, in: Die Zeit (14.06.1967). 
Bürgerkriegs fortzusetzen konnten. ${ }^{47}$ Einige von den Stipendiat:innen wurden dauerhaft Teil der intellektuellen und künstlerischen Elite der Nachkriegszeit in Frankreich, wie die Philosoph:innen Mimika Kranaki (1920-2008), ${ }^{48}$ Kostas Papaioannou (19251981), Kostas Axelos (1924-2010) und Cornelius Castoriadis (1922-1997), oder auch der Architekt und Stadtplaner Georges Candilis (1913-1995), der 1963 zusammen mit dem Architektenbüro Team-X den internationalen Architektenwettbewerb zur Bebauung des „Rostlaube“ der Freien Universität gewann als „clusterartiges Raumgefüge mit einem vernetzten System von allgemein zugänglichen Straßen und Wegen, das die Einrichtungen und unterschiedlichen Institute verbinden sollte“.49

$\mathrm{Zu}$ den Stipendiaten des französischen Staates gehörte ebenfalls der Ingenieur und Komponist Iannis Xenakis (1922-2001), der als Assistent bei Le Corbusier arbeitete und 1955 mit der Uraufführung seiner ersten Komposition Metastaseis an den Donaueschinger Musiktagen an die Spitze der internationalen Szene der Neuen Musik gelangte. Xenakis nahm 1962 an der Vortagsreihe „Musik im technischen Zeitalter“ in der Berliner Kongresshalle teil, dem Pendant zu Walter Höllerers Reihe „Literatur im technischen Zeitalter“, geleitet vom Musikwissenschaftler der Technischen Universität Hans Heinz Stuckenschmidt, und kam 1963/1964 zum „Rendezvous der musikalischen Weltkulturen in Berlin“ auf Einladung der Ford Foundation. Die Welt vom 12.03.64 berichtete von Xenakis, der in Berlin zufrieden war, da „die Herren von IBM [ihm] freundlicherweise die Arbeit mit dem Elektronengehirn der Firma“ ermöglicht haben und der unter anderem an der Bühnenmusik für die Aufführung der Hiketiden des Aischylos im Antiken Theater von Epidaurus arbeitete. ${ }^{50}$ Der Komponist durfte

47 Lucile Arnoux-Farnoux: Relations intellectuels et artistiques entre la France et la Grèce au xxe siècle : l'action de deux philhellènes, Octave Merlier (1897-1976) et Roger Milliex (1913-2006), in: Rives méditerranéennes 50 (2015), 53-64, hier: 57; vgl. dazu Nikolas Manitakis, Servanne Jollivet (Hrsg.):

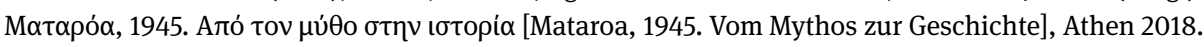
$48 \mathrm{Ihr}$ autobiographischer Roman Nationalität: Philhellene. Eine Odyssee in vierundzwanzig Briefen ist in deutscher Übersetzung bei der Edition Romiosini erschienen (übers. von Birgit Hildebrand, Berlin 2016); vgl. das Vorwort von Angela Kastrinaki, ebd., 11-27.

49 Vgl. Michael Kraus: „Kurzer Abriß der Planungsgeschichte der Rostlaube“, online abgerufen am 10. Mai 2021 auf der Webseite der Freien Universität Berlin, unter https://www.fu-berlin.de/sites/ abt-3/campus/bauprojekte/projektarchiv/seite_geschichte.html. In diesem Kontext ist ebenfalls der Architekt Ioannis Despotopoulos, auch Jan Despo (1903-1992), zu erwähnen, der nach seiner Entlassung 1946 von der Nationalen Technischen Universität Athen nach Schweden flüchtete und ab 1964 Außerordentliches Mitglied (ab 1979 Mitglied) der Akademie der Künste Berlin (West), Sektion Baukunst gewesen ist; vgl. dazu die Beiträge „Architektur und Politik: die Rolle des Architekten Ioannis Despotopoulos bei der Intensivierung der deutsch-griechischen Beziehungen in den 1960er Jahren“ von Loukas Bartatilas und „Deutsch-griechische Verflechtungen von Architekten und Stadtplanern während des Wiederaufbaus der Nachkriegszeit" von Lina Dima im Compendium der deutsch-griechischen Verflechtungen (https://comdeg.eu/compendium/).

50 Zitiert nach Stefanie Endlich, Rainer Höynck (Hrsg.): Blickwechsel. 25 Jahre Berliner Künstlerprogramm, Berlin 1988, 40. Vgl. auch den Beitrag von Wolfgang Burde: Rendezvous der musikalischen Weltkulturen in Berlin, in: ebd., 80-83. 
übrigens nicht nach Griechenland weder für die Proben noch für die Aufführung der Tragödie; dem Exilanten wurde kein Visum erteilt und sowohl der Regisseur als auch die Chorleiterin haben ihn während der Zusammenarbeit im Ausland besuchen müssen. ${ }^{51}$

In den 25 Jahren des Berliner Künstlerprogramms, die im Band von Stefanie Endlich und Rainer Höynck dokumentiert werden, kamen die ersten Einladungen nach Griechenland direkt nach dem Militärputsch vom April 1967; der Blickwechsel zeugt sowohl vom Bemühen, dem politischen Anlass Rechnung zu tragen, als auch von der schnellen Inkubationsphase des Kulturaustausches, der von Mittlerinstitutionen wie dem Goethe-Institut in Athen in Gang gesetzt wurde, dessen Rolle in der Zeit nicht genug zu betonen ist, ${ }^{52}$ wie auch von individuellen Mittler:innen in Berlin und Athen. Nicht zu unterschätzen ist die Funktion des überwiegend als Kurator in Deutschland tätigen und seit 1958 in Berlin lebenden griechischen Kunsthistorikers Christos M. Joachimides (1932-2017), dessen Ruf als Ausstellungsmacher die Joseph-Beuys-Retrospektive von 1977 oder die großen Ausstellungen im Martin-Gropius-Bau begründeten (,Zeitgeist“; „Metropolis“; „Amerikanische Kunst im 20. Jahrhundert“; „Die Epoche der Moderne“)..$^{53}$ Auch auf seine enge Freundschaft mit Peter Nestler, Leiter des Künstlerprogramms des DAAD in den 1960er Jahren, ist das Insiderwissen des Programms über die Entwicklungen in der Athener Kulturszene zurückzuführen, eine Vermittlung, die eine Reihe von Künstlereinladungen in den späten 1960er und frühen 1970er Jahren mitverursachte. ${ }^{54}$ In dieser Zeit wurden in chronologischer Reihenfolge folgende Künstler:innen bzw. Autor:innen eingeladen:

51 Siehe dazu http://www.nt-archive.gr/playMaterial.aspx?playID=198\#programs (online abgerufen am 10. Mai 2021). Der Exilant durfte erst 1975 durch ein Sondergesetz, im Rahmen des ersten „Festival Xenakis“, nach Griechenland zurückkehren.

52 Wie Eberhard Rondholz, in seinen Erinnerungen an den damaligen Programmdirektor des Athener Goethe-Instituts Johannes Weissert (1930-2006) im Heft vom 21.04.2006 der Athener Zeitschrift Anti, es treffend auf den Punkt gebracht hat, sowohl Klaus Schulz, Leiter des Instituts, als auch Johannes Weissert hatten den deutschen Kulturexport als „Export demokratischen Geistes“ verstanden. Vgl. dazu die Dokumentation von Nikoletta Dimitrouka in ihrer Masterarbeit (Neogräzistik, Freie Universität Berlin) sowie ihren Beitrag „Die deutsch-griechischen Kulturbeziehungen während der Militärdiktatur in Griechenland (1967-1974)“ im Compendium der deutsch-griechischen Verflechtungen (https://comdeg.eu/compendium/).

53 Vgl. Franziska Leuthäußer im Gespräch mit Christos M. Joachimides. Interview vom 07. März 2016, online abgerufen am 10. Mai 2021 auf der Webseite des Städel Museums unter https://cafedeutschland.staedelmuseum.de/gespraeche/christos-m-joachimides.

54 Siehe das Gespräch zwischen Christos M. Joachimides und Eleana Stoikou über die vom Goethe Institut in Athen unterstützte Ausstellung „Avantgarde Griechenland“ mit überwiegend in Paris arbeitenden Künstler:innen im Haus am Lützowplatz (Herbst 1968), anschließend gezeigt in Frankfurt und in Stuttgart, in der die künftigen DAAD-Stipendiaten Konstantin Xenakis (1931-2020), Costas Tsoclis ( $\left.{ }^{\star} 1930\right)$ und Stathis Logothetis (1925-1997) vertreten waren, online abgerufen am 10. Mai 2021 über die Webseite der Freien Universität Berlin unter https://www.cemog.fu-berlin.de/newsletter/newslet- 
Musik

- Iannis Xenakis (10.1963-09.1964)

- Theodore Antoniou (11.1967-11.1968)

- Nikos Mamangakis (01.1970-12.1970)

Bildende Künste

- Alexis Akrithakis (11.1968-12.1970)

- Konstantin Xenakis (05.1970-09.1971)

- Costas Tsoclis (02.1971-07.1972)

- Stathis Logothetis (01.1972-04.1973)

- Vlassis Caniaris (05.1973-04.1974)

- Jannis Psychopedis (05.1977-02.1978)

\section{Literatur}

- Mando Aravantinou (02.1969-05.1970)

- Alexander Skinas (04.1969-09.1970)

- Vassilis Vassilikos (08.1970-07.1971)

- Menis Koumandareas (02.1972-07.1972)

- Thanassis Valtinos (04.1974-03.1975)

- Demosthenes Davvetas (06.1985-08.1985)

- Theophilos Frangopoulos (01.1985-12.1985)

Im zweisprachigen Begleitkatalog der unter der künstlerischen Leitung von Joachimides 1986 in Athen stattgefundenen Veranstaltungsreihe „Athen in Berlin“ unterstrich der damalige Direktor des DAAD in Berlin und Leiter der Künstlerprogramms, dass die meisten der griechischen Gäste während der Militärdiktatur nach Berlin gekommen waren: „Dies gehört mit zum Selbstverständnis des Berliner Künstlerprogramms: jederzeit auch jenen Künstlern Gastfreundschaft zu gewähren, die in ihren Heimatländern der Verfolgung ausgesetzt sind“, zugleich betonend, dass , auch in ,guten Jahren“ [...] griechische Maler, Bildhauer, Komponisten und Schriftsteller gerngesehene Gäste in Berlin“ seien. ${ }^{55}$ Es ist jedoch zu konstatieren, dass diese geballte Präsenz griechischer Kultur im Berliner Künstlerprogramm sich seitdem nicht wiederholt hat.

ter-archiv/cemog-newsletter-11/wissenswert.html. Vgl. auch die Erinnerungen von Eberhard Roters an Alexis Akrithakis (1939-1994) und den „Standortnachteil“ der Künstler:innen „mit einer ungünstigen europäischen Randlage“, in: Endlich/Höynck (Hrsg.): Blickwechsel (Anm. 50), 17.

55 Wieland Schmied: Das Berliner Künstlerprogramm des DAAD, in: Christos M. Joachimides (Hrsg.): Athen in Berlin. Das Berliner Künstlerprogramm des DAAD uns seine Gäste, Athen 1986, 9-11, hier: 11. Vgl. auch Endlich/Höynck (Hrsg.): Blickwechsel (Anm. 50), 318: „,Athen in Berlin“ hieß die vom DAAD und Athener Goethe Institut organisierte Veranstaltungsreihe, die in Athen über die Arbeit von insgesamt 16 ehemaligen griechischen Gästen des Künstlerprogramms informierte. Zum Programm gehörten Lesungen, Konzerte und eine von Christos Joachimides ausgerichtete Ausstellung mit Werken von Akrithakis, Xenakis und Psychopedis sowie eigens hierfür geschaffenen Environments von Caniaris, Logothetis und Tsoclis. Die Reihe knüpfte an frühere Präsentationen dieser Art in London und Paris an“; im Kontext dieser Retrospektive, aber auch als Hervorhebung des deutsch-griechischen Kulturtransfers, dürfen auch 
Kurz vor dem Militärputsch am 21. April 1967 wurde ebenfalls Berlin nach Athen ,eingeladen“ mit der von Joachimides kuratierten Ausstellung „Berlin Berlin. Junge Berliner Maler und Bildhauer“. Diese Ausstellung, so Joachimides,

hatte das Schicksaal, die letzte demokratische Kulturveranstaltung in Athen vor dem Beginn der Militärdiktatur zu sein. Denn kaum hatten wir die Bilder auf den Weg nach Berlin gebracht, kamen die Obristen an die Macht, die jedes geistige Leben im Lande erstickten. Kurz darauf, im Juli 1967, verließ ich mit Konstantin Tsoukalas und seiner Frau Machi Griechenland. Johannes Weissert damals die Seele des Goethe Instituts in Athen - bracht uns mit seinem Wagen über einen kleinen Grenzübergang in der Nähe der Prespa-Seen in Westmazedonien nach Jugoslawien. Tsoukalas ging nach Paris, wo sich das Zentrum der griechischen Emigration zu formieren begann, und ich kam nach Berlin zurück; auf dem Höhepunkt der Studentenbewegung. ${ }^{56}$

Als Motto der Ausstellung der jungen Berliner Maler und Bildhauer ${ }^{57}$ haben die Organisatoren Michel Butors Huldigung der geteilten Stadt ausgewählt. Das Athener Publikum wurde somit konfrontiert mit Berlin als „nicht mehr nur eine ganze Stadt unter anderen, mit ihren Stadien, ihren Theatern, ihren Gerichten, ihren Museen, ihren Schaufenstern, ihren Laboratorien und ihren lärmenden Manövergeländern, Schauspielern, Sängern, Stars, Sportlern, Spezialisten, Strategen, sondern ein Horchposten, eine Versuchsbank für alle anderen Städte, sie selbst voll und ganz Theater, Zirkus, Oper, mit Kulissen und Orchesterraum, Schwelle, Schaufenster, Laboratorium“ “.58 In diesem Erwartungshorizont sind somit zum ersten Mal die Arbeiten u. a. von Baselitz, Hödicke, Koberling und Lüpertz in Griechenland rezipiert worden, sowie auch die Arbeiten eines nach Berlin migrierten griechischen Künstlers, Vagelis Tsakiridis (1936-?), der als Bindeglied zwischen der Gruppe 47 und der neugriechischen Literatur in den kommenden Jahren in Berlin eine wichtige Mittlerfunktion als Übersetzer und Herausgeber übernehmen würde.

Der Bildhauer, dem wegen Wehrdienstverweigerung die griechische Staatsbürgerschaft entzogen wurde und der seit 1963 ohne gültigen Reisepass als Staatenloser in Westberlin lebte, schrieb seit 1965 Prosa und Lyrik in deutscher Sprache. ${ }^{59}$ Er wurde zum

die Einladungen von 1985 verstanden werden, des in Paris lebenden Autors, Essayisten, Kulturkritikers und Malers Demosthenes Davvetas ( $\left.{ }^{\star} 1955\right)$, der in Joseph Beuys seinen Mentor sieht, und des Generalsekretärs des griechischen PEN-Clubs, Übersetzers deutscher Literatur ins Griechische und im Antidiktaturkampf engagierten (s. unten Anm. 69) Schriftstellers Theophilos Frangopoulos (1923-1998).

56 Christos M. Joachimides: Athen und Berlin 1967-74, in: ders. (Hrsg.): ebd., 13.

57 Diese Ausstellung der Deutschen Gesellschaft für Bildende Kunst (Kunstverein Berlin) in Zusammenarbeit mit dem Goethe-Instituts Athen wurde im Zapeion in Athen gezeigt (24. Februar bis 19. März 1967) und mit einem schlichten Katalog dokumentiert; sie, so Joachimides, in seinem Geleitwort, ,ist kein Programm, zieht keine Bilanz, propagiert keine neue ,Richtung‘. Sie ist lediglich das Zeugnis einer Generation, die sich selbst im Erlebnis einer neu gewonnenen Freiheit darstellt. “ Berlin Berlin. Junge Berliner Maler und Bildhauer, Berlin 1967, [9].

58 Ebd., [7].

59 Ein Porträt des Autors bietet das Nachwort von Klaus Roehler in Vagelis Tsakiridis: Hallelujah! 
letzten Treffen der Gruppe 47 in der „Pulvermühle“ eingeladen, erhielt für seine Lesung einen Anerkennungspreis, wurde von Hans Werner Richter „nach vorn“ geschoben, „ganz nach vorn, bis an den Preis der Gruppe 47“ als eine „politische Demonstration [...] für ein freies Griechenland“.$^{60}$ Nach dem Militärputsch engagierte sich Tsakiridis in der Übersetzung und Vermittlung der neugriechischen Literatur in den späten 1960er Jahren; dem Tsak verdankt Vassilis Vassilikos die Übersetzung und deutsch-deutsche Zirkulation seines Romans $Z$ - Anatomie eines politischen Mordes, erschienen auf beiden Seiten der Mauer: 1968 im Westen bei Blanvalet Berlin in der damals konzipierten Reihe „Die Griechen“ und 1970 als Lizenzausgabe im Osten beim Verlag Neues Leben Berlin. ${ }^{61}$

Mit dem Namen von Tsakiridis sind viele weiteren Initiativen des (literarischen) Transfers zwischen Griechenland und Deutschland unter dem Vorzeichen des antidiktatorischen Kampfes verbunden. ${ }^{62}$ Für die achte Lieferung von Luchterhands Lose-

71 Protokolle, Neuwied/Berlin, 1968, 49-51. Mit der Ausnahme einer Athener Gedichtsammlung von

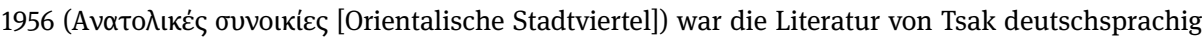
(siehe etwa u.a.: Gedichte für die Jungfrau am Brunnen und Prosa, Berlin/Neuwied 1967; Tsak's Zacke, Darmstadt 1973); 1969 hat er in Düsseldorf die Anthologie Super Garde. Prosa der Beat- und Pop-Generation herausgegeben. Als Alexis Pappas hat er ferner beim Verlag Blanvalet in Berlin die Romane Nachtcafé (1969) und Schaltjahre (1970) veröffentlicht. Ob Tsakiridis 2001 noch lebte, konnte Vassilis Vassilikos in seiner Rede in Frankfurt, als Griechenland Gastland der 53. Frankfurter Buch-

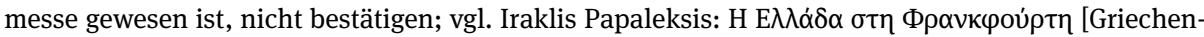
land in Frankfurt], in: $\Delta \iota \alpha \beta \dot{\gamma} \zeta \omega$ [Lesen] 420 (Juli-August 2001), 28-37, hier: 32.

60 Hans Werner Richter: Mittendrin. Die Tagebücher 1966-1972, hrsg. von Dominik Geppert in Zusammenarbeit mit Nina Schnutz, München 2012, 90, Eintrag vom 25.10.1967.

61 Der Druckgenehmigungsvorgang für die ostdeutsche Lizenzausgabe des $Z$ (Verlag Neues Leben, Bundesarchiv, Signatur DR 1/3543a) verrät ambivalente Bedenken bezüglich das Werks eines Autors, der „kein Marxist“ sei und deswegen die „Frage nach dem Ausweg [...] offen, zu offen [...]“ im Roman bliebe, den ein „deutlich zu vernehmender Ton der Resignation beherrscht“ (320). Obwohl der Gutachter die Veröffentlichung empfiehlt, erwägt er zudem die Option eines Vorwortes, das „den notwendigen Zugang zu Vassilikos und das richtige Verständnis“ seines politisch-historischen Standortes verschaffen sollte (321). Im Gegensatz dazu ist die zweite Gutachterin der Ansicht, „dass ein erklärendes Nachwort nicht notwendig“ sei, da das Buch „keine falschen Akzente“ setzte (324), wobei ein dritter Gutachter empfiehl, den Roman in einem anderen Verlag erscheinen zu lassen, ,der einen größeren Leserkreis anspricht“ (331). $Z$ blieb beim Verlag Neues Leben, jedoch ohne Vor- bzw. Nachwort. 62 Siehe z. B. das Vorwort Aris Fakinos u. a. (Hrsg.): Schwarzbuch der Diktatur in Griechenland. Eine Dokumentation, Reinbek bei Hamburg 1970, 7-9, hier: 7, in der aus dem Französischen übersetzten Dokumentation über Griechenland der Wochenzeitschrift Athènes-Presse Libre von Aris Fakinos, Clément Lépidis und Richard Soméritis; unverkennbar ist die Perspektive des ,dreißigjährigen Krieges‘: „Es ist in der Tat erstaunlich, dass dieses Volk noch nicht kapituliert hat, denn das griechische Volk kämpft nicht erst seit drei Jahren, sondern seit dreißig für seine Freiheit. Dieser Kampf wurde in Europa völlig ignoriert. Was in Griechenland während der letzten Jahrzehnte geschah, interessierte kaum jemanden im Lager der sogenannten ,freien Welt‘, allenfalls Nato-Strategen, alternde Archäologen und sonnensüchtige Touristen. Während man mit ausländischer ,Hilfe‘ Raketenbasen baute, Museen restaurierte und Küsten mit Hotels besetzte, musste das kleine, auch ,stolz' genannte Volk die Schlinge um seinen Hals spüren.“ 
blatt Lyrik hat er etwa sieben Gedichte aus dem Griechischen gegen die Militärdiktatur übersetzt, ${ }^{63}$ für die Akzente das der neugriechischen Literatur gewidmete Heft 2 (1968) herausgegeben, ${ }^{64}$ am Projekt von Paul Dessau „Für Mikis Theodorakis“ teilgenommen, bei dem der Wortlaut des Verbots von den Obristen der Musik von Theodorakis vertont wurde. Und nicht zuletzt, Tsakiridis hat mit der Übertragung von Jannis Ritsos' Gedichten in der Reihe Quarthefte des Verlags Klaus Wagenbach in Berlin die bundesrepublikanische Rezeption des auf Leros verbannten Dichters initiiert. ${ }^{65}$ Der Übersetzer und der Verleger hatten folgende Notiz vorangestellt:

Der Außenminister der griechischen Putschisten, Pipinelis, wird diese erste deutsche Ausgabe einer der großen Lyriker seines Landes wahrscheinlich auch für „einen Missbrauch des freiheitlich-demokratischen Bodens der Bundesrepublik für eine nicht objektive Berichterstattung über die Situation in Griechenland“ halten; ein solcher Missbrauch „erweckte schmerzliche Gefühle“ in ihm, am 2. August 1968.

Die Bundestagsabgeordneten Huys, Toussaint, Stecker (CDU) und Ertl, Kubitza, Zoglmann (FDP) erklärten am gleichen Tag, nach ihrem Aufenthalt in Griechenland, man habe den Besuch von Straflagerinseln nicht für nötig gehalten, da „genügend Berichte über eine zufriedenstellende Behandlung der Gefangenen“ vorlägen. $\mathrm{Zu}$ diesen Berichten gehört jedenfalls nicht der von Amnesty International über die angewandten „Techniken der Folter“ gegenüber den (mindestens) 2800 Gefangenen. Wir fordern die Bundestagsabgeordneten auf, bei ihrer nächsten vom griechischen Informationsministerium bezahlten Reise nach Jannis Ritsos, inhaftiert auf Leros, zu fragen.

Bis heute hat Ritsos nicht einmal die Erlaubnis zu schreiben.

Mit diesem Rezeptionsvorgang wurde es allmählich möglich, den Namen Jannis Ritsos, wie auch die Namen weiterer griechischen Autor:innen genau auszusprechen, was am 9. Dezember 1967 noch nicht der Fall war. An jenem Abend hatte Hans Werner Richter zu einem Treffen der Gruppe 47 in der Erdener Str. 8 mit griechischen Autor:innen in der Emigration eingeladen, aufgenommen für das III. Programm des Südwestfunks unter dem Titel Die Gruppe 47 und die exilierte griechische Literatur. Bericht über ein Treffen: ${ }^{66}$

63 Es handelt sich um Texte von Manolis Anagnostakis (1925-2005), Giorgos Gavalas (1922-2018), Michalis Katsaros (1919-1998), Titos Patrikios (`1928), Jannis Ritsos, Vassilis Vassilikos und Vassilis Ziogas (1937-2001).

64 Mit der Ausnahme zweier Gedichte von Kavafis sind im Heft eine damals in Athen lebende Dichterin (Eleni Vakalo, 1921-2001) sowie zehn Dichter vertreten, davon vier in Athen (Giorgos Seferis, Odysseas Elytis, Aris Diktaios, 1919-1983 und Jannis Dallas, 1924-2020), zwei in Verbannung (Jannis Ritsos und Giorgos Gavalas) und vier im Exil (Dimitris Christodoulou, 1924-1991; Titos Patrikios; Michalis Katsaros; Vassilis Vassilikos).

65 Vgl. zu den Wegen der Rezeption von Ritsos im deutschsprachigen Raum die Dissertation von Maria Biza: Deutsch-griechischer Lyriktransfer im 20. Jahrhundert. K. Kavafis, G. Seferis und J. Ritsos in deutschen Übersetzungen, Bearbeitungen und Anthologien, München 2017, Kap. 3.2.2 und 3.3.

66 Hauptabteilung Information, Dokumentation und Archive des SWR und SR, SWR-BAD 67622, Erstausstrahlung am 06. Juni 1968. Die Regie führte Dieter Ehlers. 
[Stimme off] 9. Dezember 1967. In der Erdener Str. 8 in Berlin treffen sich Schriftsteller der Gruppe 47 mit griechischen Kollegen, Verlegern, Regisseuren, Journalisten, Malern, die das Regime verfolgt. Sie kommen aus Paris, London, München, Berlin. Eingeladen hat Hans Werner Richter.

[Hans Werner Richter] Wir haben ein bisschen später anfangen müssen, weil ... weil Tsakiridis vergesslich die griechischen Texte vergessen hat, was ja nicht unwichtig ist bei literarischen Lesungen. Außerdem habe ich Häutung, wenn ich heute den ganzen Abend damit spiele. [Er zeigt ein Komboloi.] Titos Patrikios, Rechtanwalt und Lyriker, das gibt's, Rechtsanwalt und Lyriker, aus Athen, hat mir heute diese Kette aus Paris mitgebracht. Ich habe in Athen und Saloniki gesehen, dass alle Männer mit diesen Ketten spielen in Griechenland. Und ich habe von da ab, das ist etwa sechs Jahre her, immer mit meinem Autoschlüssel gespielt, und habe immer so eine Kette haben wollen. [...] Ich bin der Meinung, dass wir etwas mehr für die emigrierte oder vertriebene oder exilierte griechische Literatur tun sollten als wir bisher getan haben. Denn ich habe den Eindruck, wir gewöhnen uns immer sehr schnell daran, dass irgendwo eine Diktatur existiert, und so weiter. Dazu habe ich Sie also eingeladen. Es werden einige griechische Schriftsteller lesen, griechisch, und dann werden deutsche Schriftsteller die deutschen Texte lesen. Zuerst liest Lila Maraka, und zwar liest sie ein Gedicht eines, des berühmtesten, wie man mir sagt, ich weiß nicht gut Bescheid, griechischen Lyrikers, Jannis Ristos.

[Lila Maraka] Ritsos

[Hans Werner Richter] Ristos

[Lila Maraka] Ritsos

[Hans Werner Richter] Jannis Ristos

[viele Stimmen] Jannis Ritsos

[Hans Werner Richter] Frau Maraka, bitte. Also, es liest Jannis Ristos.

[Lila Maraka] Ritsos

[Hans Werner Richter] Ritsos. Wenn ich nicht alle griechischen Namen genau ausspreche, nehmen Sie es mir nicht übel. Ich kenne mich nicht gut aus.

In seinem Tagebuch hat Hans Werner Richter seinen unmittelbaren Eindruck von jenem Abend dokumentiert; unverkennbar bleibt auch hier der leicht orientalisierende Ton:

Am Sonnabend hier in Berlin ein Abend für ein freies Griechenland, an dem etwa ein Dutzend griechische Schriftsteller teilnahmen. Sie kamen fast alle aus Paris. Begabt, emotionell, ideologisch. Es nahmen etwa hundert Leute hier in der Wohnung daran teil: Günter Eich, Renate Rasp, Jürgen Becker, Reinhard Baumgart, Günter Herburger, Tankred Dorst, Peter Wapnewski. Es lasen zwei griechische Schriftsteller: Patrikios und Vassilikos. Bei allen eine seltsame Mischung: Nationalismus und Kommunismus, die griechische Erde und die Weltrevolution. Die Diskussion ergab nichts, nur Ausrufe, Proteste, Reden, Reden. Nach kurzer Zeit waren alle miteinander zerstritten, alle Griechen, ein merkwürdiger Eindruck, ein Caféhaus aus Athen in meiner Wohnung. ${ }^{67}$

67 Richter (Anm. 60), 94. Mein herzlicher Dank gilt Helga Neumann (Literaturarchiv der Akademie der Künste, Berlin) für den Hinweis auf diese Tagebuchstelle. Die starke Disharmonie in der griechischen Emigration wird auch im Brief von Tsakiridis an Hans Bender bestätigt: „Viele Griechen waren gestern in Berlin - wenig hatten sie zu sagen. Veraltete Revolutionär[e] der alte[n] Schule, die mich an Väter erinnerten, die für ihre Söhne ständig eine Tracht Prügel bereit haben, [V]erbesserer einer Welt derer innere[n] Krankheiten sie nicht ahnen und sie nur zu kämmen versuchen. Ich bin für sie natürlich ein Faschist, ,du versuchst mit de[n] griech. Gefangene[n] einen Namen zu machen‘ sagte 


\section{Der Stichtag 1967 und Walter Höllerer}

Damit die bundesrepublikanische (literarische) Öffentlichkeit einen angemessenen Eindruck vom zeitgenössischen Griechenland und dessen intellektueller Produktion bekommen konnte, waren mehrere Initiativen des Kulturtransfers in den 1967 sich anschließenden Jahren notwendig, deren detaillierte Schilderung die „Berliner Weltliteraturen“ ausklammern und den Rahmen sprengen würde. Der Neustart des intellektuellen Widerstandes in Griechenland nach der Aufhebung der Präventivzensur im Herbst 1969 hat sich in einer Reihe von Publikationen niedergeschlagen, beginnend mit der emblematischen Anthologie 18 Kci $\mu \varepsilon v \alpha$ [18 Texte] von 1970 und den darauf-

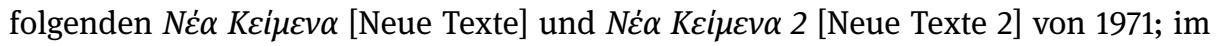
Vorzeichen einer demokratischen Einheit jenseits der Fronten des „dreißigjährigen Krieges“ bot hiermit der linksgerichtete Verlag Kedros ein Dach für Autor:innen und Intellektuelle aus dem gesamten antidiktatorischen Spektrum, von Seferis bis Ritsos, ohne die Ideologie zum Dreh- und Angelpunkt zu machen. ${ }^{68}$

Diese neue Konstellation ermöglichte auch das für die deutsch-griechischen literarischen Verflechtungen zentrale Heft 2/1971 der Akzente, das zweite der neugriechischen Literatur gewidmeten Heft der Zeitschrift; dessen Konzeption begann mit der Einladung, die Hans Bender 1968 vom Goethe Institut erhielt, einen Vortrag über politische Dichtung in Athen und in Thessaloniki zu halten. In enger Zusammenarbeit mit Johannes Weissert und den damals ihm in Griechenland vertraut gewordenen Schriftsteller:innen, mit allen jenen ,die mich über die Situation unterrichtet, die mir Gedichte der Verbannten in die Tasche gesteckt, die mich auf diese Texte aufmerksam gemacht oder sie übersetzt haben“, ${ }^{99}$ hat Bender zum Ziel des Heftes erklärt, „die

mir sogar einer. Es ist traurig, nicht wahr? Die Welt wird nicht durch Bomben, sondern durch $100 \%$ Idiotie zu Grunde gehen - aber darüber sind wir, glaube ich, einig. “ Sammlung Akzente Korrespondenz, Literaturarchiv Sulzbach-Rosenberg, Signatur 01AK/22750.

68 Als zentraler Moment des deutsch-griechischen antidiktatorischen Kulturtransfers gilt die Anthologie mit Beiträgen aus diesen drei Publikationen und weiteren Essays gegen die Militärdiktatur, die, herausgegeben von Danae Coulmas (*1934), Redakteurin damals beim Griechischen Programm der Deutschen Welle in Köln, auf Deutsch bei Fischer Verlag erschienen ist: Die Exekution des Mythos fand am frühen Morgen statt, übers. von Danae Coulmas, Nonna Nielsen-Stokkeby und Georg Walther Heyer, Frankfurt a. M. 1973 (siehe die Neuausgabe bei der Edition Romiosini: Die Exekution des Mythos, Berlin 2017). Vgl. zur Rolle der Deutschen Welle die Dokumentation der Veranstaltung des Centrum Modernes Griechenland der Freien Universität Berlin „Gemeinsamer Widerstand: die Obristendiktatur in Griechenland und die deutsche Öffentlichkeit“ (20. Juni 2014), online abgerufen am 10. Mai 2021 auf der Webseite der Freien Universität Berlin unter https://www.cemog.fu-berlin.de/ aktivitaeten/mediathek/gemeinsamer-widerstand-1974/index.html.

69 Hans Bender: Zu diesem Heft, in: Akzente 11 (1971), 97-98. Neben zwei Aufsätzen von Wolfgang Rieger und Anselm Haverkamp sind im Heft zwei Autorinnen (Kay Cicellis, 1926-2001, und Maria Kyrtzaki, 1948-2016) und dreizehn Autoren (Theophilos Frangopoulos, Giorgos Seferis, Jannis Ritsos, Manolis Anagnostakis, Rodis Roufos, 1924-1972, Andreas Lentakis, 1935-1997, Titos Patrikios, Nikos 
Literatur und ihr Verhalten innerhalb Griechenlands“ zu zeigen und, ohne die eine Gruppe gegen die andere auszuspielen, die Autor:innen im Exil auszuklammern, sowie zugleich die Frage zu beantworten, warum die „deutsche Interpretation griechischer Literatur veraltet oder verlogen" ${ }^{\text {"70 }}$ ist.

Nahm der einleitende Essay von Theophilos Frangopoulos „Die Militärregierung und die Literatur“ zum ersten Schwerpunkt Stellung, versuchte der Aufsatz des Konstanzer Literaturwissenschaftlers und damaligen DAAD-Lektoren in Irland, Anselm Haverkamp, die zweite Frage zu beantworten. ${ }^{71}$ Anknüpfend an die hier schon zitierte Polemik Helmut Salzingers gegen die institutionalisierte Selektion der neugriechischen Literatur in der BRD, etwa die „nichtssagenden Scheininformationen“ von Isidora Rosenthal-Kamarinea (1918-2004), die das Fach der byzantinischen und neugriechischen Philologie an der Universität Bochum vertrat, die die Redaktion der Zeitschrift hellenika der Vereinigung der Deutsch-Griechischen Gesellschaften innehatte und somit als Kontrollinstanz des Transfers fungierte, ${ }^{72}$ bzw. die politischen „Affinitäten zwischen dem konservativen Griechenland und dem bundesrepublikanischen Deutschland der Adenauer-Ära“ im

Eggonopoulos, 1907-1985, Markos Avgeris, 1884-1973, Thanassis Valtinos, Stratis Tsirkas, Pavlos Papassiopis, 1906-1977, Giorgos Ioannou, 1927-1985) vertreten. Im Literaturarchiv Sulzbach-Rosenberg lässt sich aus dem dort bewahrten Briefwechsel die Geschichte dieses Heftes minutiös rekonstruieren, auch im Kontext des erbitterten Streits, der ausgebrach „zwischen der äußeren Emigration, vertreten durch Herrn Skinas, und der inneren, zu deren Sprecher in diesem Fall sich anscheinend unser Freund Frangopoulos gemacht hat“. Siehe den Brief von Weissert an Bender vom 08. August 1970, Sammlung Akzente Korrespondenz, Literaturarchiv Sulzbach-Rosenberg, Signatur 01AK/24112; vgl. dazu Endlich/Höynck (Hrsg.): Blickwechsel (Anm. 50), 98. Im Brief von Frangopoulos an Bender vom 31. Juli 1970 wird Weissert „als Träger unserer Idee und Pläne“ und „Bevollmächtigter“ dargestellt: „Und wie gesagt, ist unserer ,Spokesman“ Herr Weissert allein.“ Ebd., Signatur: 01AK/13906; die Tatsache, jedoch, dass Johannes Weissert auf die Nennung seines Namens als Co-Editor des Heftes vorerst verzichtet hat, zeigt, dass er nur zu gut wusste, „wie sehr seine Arbeit im Goethe-Institut der Deutschen Botschaft ohnehin missfiel, sein Engagement für linke bzw. verbotene Literatur, so fürchtete er nicht zu Unrecht, hätte seine Position zusätzlich gefährden können.“ Eberhard Rondholz: Die Junta und die Deutschen. Zwischen Realpolitik und Solidarität - Eine Bilanz 50 Jahre nach dem Putsch, in: Hellenika NF 12 (2017), 20-46, hier: 30.

70 Bender, ebd., 97 und 98.

71 Anselm Haverkamp: Griechische Literatur in Deutschland. Bemerkungen zu den Bedingungen der Rezeption neugriechischer Literatur in Deutschland, in: ebd., 173-189. Der Aufsatz, versehen mit einem Motto aus Horkheimers und Adornos Dialektik der Aufklärung: „Nicht um die Konservierung der Vergangenheit, sondern um die Erlösung der vergangenen Hoffnung ist es zu tun“, erschien ursprünglich 1970 in der in Zürich herausgegebenen antidiktatorischen Zeitschrift Propyläa von Argyris Sfountouris, der für das Akzente-Fassung ebenfalls die Überstzungen der Gedichte von Seferis, Ritsos und Anagnostakis lieferte; Bender druckte 1971 eine erweiterte Fassung. Sammlung Akzente Korrespondenz, Literaturarchiv Sulzbach-Rosenberg, Signaturen 01AK/15262 und 01AK/15263.

72 Isidora Rosenthal-Kamarinea, die für das Akzente-Heft die Übersetzungen von Kyrtzaki und Avgeris geliefert hatte, konterte mit ihrem Beitrag im Heft 4 (1971): Der wahre Sachverhalt. Zu Anselm Haverkamps Aufsatz „Griechische Literatur in Deutschland“ in Akzente 2/71, in: Akzente 18 (1971), 379-383. Zur Vermittlerin Isidora Rosenthal-Kamarinea vgl. Pechlivanos (Anm. 19), 362-368. 
reaktionären Griechenlandbild der Reiseliteratur des Publizisten Johannes Gaitanides, ${ }^{73}$ gab sich Haverkamp nicht zufrieden mit einer Kritik der Abwesenheit politischer wie sozialer Thematik, sondern sah in der kritischen Aufhellung der Bedingungen der Rezeption und der Bestimmung der Grundzüge des deutschen Griechenlandbildes die Chance, nach dem Stichtag 1967, Griechisches aus einer neuen Perspektive zu lesen.

Die ideologiekritische Pointe, gerichtet sowohl an die deutsche als auch an die griechische Literatur und Literaturgeschichte, betraf das Abbauen des Vorurteils über eine Besonderheit Griechenlands, die mit der Seele zu suchen und zu finden ist in der Mythisierung von Geschichte und Volkscharakter und im Primat einer Landschaft, die Zeit und Geschichte negiert. Im Gegensatz zu einem vom starken Licht geblendeten Griechenland sei eine Kritik nötig, wie sie etwa früh der Marxist Kostas Varnalis in seinem Essay „Die fremden Einflüsse in unserer Literatur“ betrieben hatte, die Autarkie griechischer Literatur als Fiktion entlarvend und Roland Barthes’ Ausführungen zum Mythos als entpolitisierte Aussage vorwegnehmend. ${ }^{74}$ Das Plädoyer, da auch nach 1967 das Griechenlandbild keine Veränderungen im Sinne einer Revision erkennen ließ, war „zugunsten einer realen Einschätzung [Griechenlands] politischen, wirtschaftlichen und geistigen Existenz“, jenseits jener in Haverkamps Augen ärgerlichen Wiederkehr des Mythos eines universalen Griechentums in esoterischer Abmachung, etwa in den 1968 und 1969 von Günter Dietz (1930-2017) herausgegebenen Übersetzungen der Sechzehn Haikus von Seferis und der gefeierten Griechenlandhymnik des künftigen Nobelpreisträgers Odysseas Elytis von 1979 To Axion Esti - Gepriesen sei. ${ }^{75}$

Ob dieser Aufsatz nicht nur Hans Bender sondern ebenfalls Walter Höllerer „sehr angesprochen hat" ${ }^{76}$ lässt sich aus den vorhandenen Archivalien nicht rekonstruieren. Die höfliche Absage, die 1964 Günter Dietz auf seine Anfrage bekommen hat, Übersetzungen von Seferis und Elytis in den Akzenten zu veröffentlichen, ist ein Indiz, auch weil damit der Wille der Zeitschrift und analog des LCB offenkundig wird, „den anderen zwei Schritte voraus“ zu sein:

Herr Professor Höllerer und ich haben Ihre Übersetzungen hin und her gereicht und jetzt erst darüber entschieden. Die Texte von Elytis und von Sepheris sind eine Veröffentlichung wert - jedoch nicht unbedingt in einer Zeitschrift, die am liebsten den anderen zwei Schritte voraus sein möchte. Sepheris - Sie können es sich vorstellen - wurde nach dem Bekanntwerden des Nobelpreises in

73 Haverkamp (Anm. 71), 175 und 180-181. Vgl. dazu oben, Anm. 19.

74 Den Aufsatz von Varnalis hatte Danae Coulmas ins Deutsche übersetzt und literarhistorisch kontextualisiert, vgl. Danau Coulmas: Ein marxistisches Plaidoyer für die Idee der Weltliteratur, in: Poetica 2 (1968), 88-91 und 92-99.

75 Haverkamp (Anm. 71), 189 und 185. Zum Mittler Günter Dietz vgl. Andrea Schellinger: Bemerkungen zum übersetzerischen Werk von Günter Dietz (1930-2017), in: Exantas. Eine griechisch-deutsche Kulturzeitschrift 31 (Juli 2020), 38-43.

76 Brief von Bender an Haverkamp vom 16. Februar 1971, Sammlung Akzente Korrespondenz, Literaturarchiv Sulzbach-Rosenberg, Signatur 01AK/15262. 
allen Zeitungen und Zeitschriften gedruckt; Elytis fand, zumindest bei den Lyrikkennern Aufmerksamkeit, als Hilty seinen Band herausbrachte. Hinzu kommt, dass wir tatsächlich für die nächsten vier AKZENTE-Hefte eine feste Planung haben. ${ }^{77}$

Für eine Auseinandersetzung Höllerers mit der neugriechischen Literatur aus seiner polykontextural ansprechbaren und mehrfachinkludierten ,Adresse‘, dem kommunikativen Knoten eines Modernisierers in der institutionellen und medialen Konfiguration der 1950er und 1960er Jahre, ${ }^{78}$ gibt es vor den DAAD-Einladungen der späten 1960er Jahre spärliche Zeugnisse. Ein früher Aufsatz über Konstantinos Kavafis zeigt auf jeden Fall, dass er in der neugriechischen Literaturgeschichte und der Kavafisphilologie bewandt gewesen ist und Kavafis’ Leistung vor der „Entstehung einer modernen und relativistischen Einstellung zur Zeit und zur Vergangenheit in der Gegenwart“ zu würdigen trachte. ${ }^{79}$ Seine Buchbesprechung der Erzählungen Flut und Ebbe von Kay Cicellis - der in Marseille geborenen und damals noch nur auf Englisch schreibenden griechischen Autorin, deren Romane in den 1950er Jahren u. a. Annemarie und Heinrich Böll ins Deutsche übersetzten - verrät zugleich sein Interesse, die Erzählungen in diesem ungewöhnlichen Buch, als Antwort auf die Fragestellungen, „die sich in der Kunst wie in der Dichtung unserer Tage zugleich anbieten: die Frage nach der Entgegensetzung und der Verbindung von Abstraktion und Gegenständlichkeit“, Ontologie und Psychologie in einer Weltentdeckung, „wie sie nur auf den Klippeninseln in Flut und Ebbe des südlichen Meeres in solcher Art konzipiert werden konnte““ 80

Dieser Wille, jene Autor:innen auch in Griechenland zu entdecken, die wie Kay Cicellis vieles von dem ansprechen, „was heute die junge Dichtung beschäftigt“, und somit eine, wie Haverkamp über das $Z$ von Vassilis Vassilikos schrieb, „neue Einstellung zum gegenwärtigen Griechenland, vermittelt [...] durch Solidarität" ${ }^{\text {81 }} \mathrm{zu}$ gewinnen, informiert m. E. die Einladungen aus Griechenland im Berliner Künstlerprogramm der späten 1960er und 1970er Jahre. Weder Elytis noch, nach 1974, Ritsos wurden eingeladen, sondern Autor:innen der extrovertierten Nachkriegsgeneration, wie die Joyce-Übersetzerin, Dichterin und Mitarbeiterin der neoavantgardistischen Zeitschrift Пádı [Wieder] Mando Aravantinou (1926-1998); der Mitarbeiter des Griechischen Programms der Deutschen Welle in Köln Alexander Skinas (1924-2012), dessen

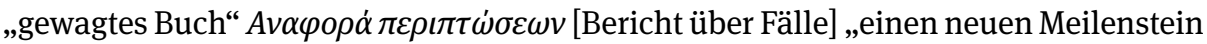

77 Brief von Bender an Dietz vom 13. März 1964, ebd., Signatur 01AK/12205.

78 Michael Peter Hehl: Berliner Netzwerke. Walter Höllerer, die Gruppe 47 und die Gründung des Literarischen Colloquiums Berlin, in: ders., Achim Geisenhanslüke (Hrsg.): Poetik im technischen Zeitalter: Walter Höllerer und die Entstehung des modernen Literaturbetriebs, Bielefeld 2013, 155-189, hier: 158 und 172.

79 Das Typoskript mit dem Titel „Über Konstantinos Kavafis“ ist verfügbar im Vorlass Walter Höllerer im Literaturarchiv Sulzbach-Rosenberg, Signatur 02WH/154/1.

80 Walter Höllerer: Kay Cicellis. Flut und Ebbe, in: Weltstimmen 6 (1953), 269-272, hier: 269 und 270.

81 Haverkamp (Anm. 71), 189. 
in der Literatur Griechenlands“ gesetzt habe;2 der ,Neorealist“ Menis Koumandareas (1931-2014), vorgeschlagen für das Künstlerprogramm von Vassilis Vassilikos, u. a. während der 1960er Jahre Übersetzer von Hermann Hesse, Carson McCullers und William Faulkner; und nicht zuletzt Thanassis Valtinos (`1932), der in der dialogischen und polyphonen Erzählung Der Marsch der Neun, einziger neugriechische Text in der Reihe der LCB-Editionen des Literarischen Colloquiums in der Übersetzung von Johannes Weissert, mit der Herstellung einer fiktiven Zeugenschaft über den Bürgerkrieg das Narrativ des griechischen „dreißigjährigen Krieges“ als Zusammenprall von Heiligen und Dämonen hinterfragt. ${ }^{83}$

Ob Walter Höllerer nach Griechenland gereist ist und in Athen, wie Hans Bender, die Bekanntschaft der Literaturszene nach dem Militärputsch 1967 oder 1968 gemacht hat, ist nach dem jetzigen Stand meiner Forschungen nicht zu konstatieren. Die Einladung nach Athen wurde vom Leiter der Athener Zweigstelle des Goethe Instituts schon im März 1966 ausgesprochen und nach der Zusage Höllerers im August 1966 wurden seine Vorträge mit der vom Börsenverein des Deutschen Buchhandels geplanten Ausstellung avantgardistischer moderner deutscher Literatur für den Herbst 1967 zusammengelegt:

Wir versuchen hier in Griechenland etwas systematisch das vorhandene Interesse an unserer Literatur weiter zu wecken und zu kultivieren. Im Oktober d. J. wird Marcel Reich-Ranicki kommen, im Frühjahr dann Professor Jens. Ich hoffe auch, dass wir im März noch die Kafka-Ausstellung

\footnotetext{
82 So der erste Eindruck von Mando Aravantinou in ihrer Buchbesprechung der griechischen Erstausgabe von 1966 in: Books Abroad 42/2 (1968), 319-320, hier: 320. Der an der deutschen Teilübersetzung des Bandes bei Suhrkamp beteiligte Schriftsteller Wolfgang Maier, der bei Walter Höllerer studiert hat und Mitarbeiter bei den Zeitschriften Akzente und Sprache im technischen Zeitalter sowie Mitglied der Gruppe 47 war, schrieb in seinem Nachwort: „Skinas, mit fremden Sprachen und Literaturen vertraut, während er immer noch der eigenen Sprache nachspürt, hat einen Ausbruch gewagt, ohne seine Basis, Geschichte und Herkunft, zu verlassen. Die ungewöhnliche Form des Zerbrechens, Zusammenfügens, Zerbrechens bedeutet gleichzeitig Abwehr und Annahme der Tradition, eine Beziehungssucht, jedes mit jedem in Verbindung zu bringen, die sich durch das ausgeklügelte Schema seiner Texte hindurchzieht, ohne dass es zum herkömmlichen, harmonisierenden Bezug kommt, deutet auf ein ,gestörtes' Verhältnis zur polychromen Masse der griechischen Kultur, einen zur Eruption bereiten Solipsismus, in Kunst umgemünzt, der in der modernen Literatur nicht vereinzelt dasteht.“ Wolfgang Maier: Statt eines Nachworts, in: Alexander Skinas: Fälle. Prosa, übers. von Richard Kruse, Frankfurt a. M. 1969, 147-148, hier: 148.

83 Walter Höllerer (Hrsg.): Autoren im Haus. Zwanzig Jahre Literarisches Colloquium Berlin, Berlin 1982, 276. Vgl. zu den Kontroversen, die das späte Werk von Valtinos über den griechischen Bürgerkrieg in der literarischen Öffentlichkeit verursacht hat: Joachim Winkler: Dimitris Raftopoulos und die „Schwarze Literatur“. Zum erinnerungskulturellen Widerstands- und Bürgerkriegsdiskurs der griechischen Nachkriegsliteratur, Berlin 2018, Kap. 5.1-5.6. In der Bibliothek Edition Romiosini siehe auch: Thanassis Valtinos: Abstieg, Flussaufwärts. Ausgewählte Prosa, übers. von Ulf-Dieter Klemm, Hans Eideneier, Danae Coulmas und Andrea Schellinger, Berlin 2017 und ders.: Brief an Christa Wolf, übers. von Ulf-Dieter Klemm, Berlin 2017, sowie: Hommage à Thanassis Valtinos, Berlin 2017.
} 
von Herrn Dr. Wagenbach zeigen können, nachdem wir im November letzten Jahres die ThomasMann-Ausstellung mit gutem Erfolg zeigten. [...] Die Ausstellung avantgardistischer moderner deutscher Literatur, die ich mit Herrn Taubert plane, soll in erster Linie das Ziel verfolgen, dass die hier interessierten literarischen Kreise auch mit der jüngsten Produktion vertraut gemacht werden, um dann hoffentlich im Rahmen Ihres Besuches ein Kolloquium zwischen einigen deutschen und griechischen Schriftstellern für das Jahr 1968 vorzubereiten. [...] Als Termin für Vortrag und Ausstellung würde von uns aus gesehen der November 1967 besser passen als der Oktober. ${ }^{84}$

Auch wenn im September 1967 das Projekt vom Auswärtigen Amt nach Schulz „scheinbar genehmigt ist“", die Einladung sogar für den Februar 1968 wiederholt wurde, ${ }^{85}$ ist „gleich im ersten Jahr der Diktatur eine Ausstellung avantgardistischer Literatur ,einstweilen' verschoben, da es der Botschaft ,untunlich erschien, amtliche Stellen durch provokatorische Themen zu irritieren““ “. ${ }^{86}$ Wie dem auch mit der Frage sei, ob Höllerer schon in Athen oder erst in Berlin die erste ,artist in residence“ aus Griechenland, die Dichterin Mando Aravantinou kennenlernte - die 1969 in Westberlin mit dem Typoskript der französischen Übersetzung ihrer 1962 und 1964 herausgegeben Bände $Г \rho \alpha \varphi \eta \dot{~} A$ [Schrift A] und $\Gamma \rho \alpha \varphi \dot{\eta} B$ [Schrift B] ankam in der Gewissheit, dass ein Rückflug von Berlin nach Athen das Unwahrscheinlichste wäre ${ }^{87}$ - sowohl in Höllerers Nachlass als auch im Aravantinous Berliner Tagebuch, verfasst nach dem Vorbild von Witold Gombrowicz

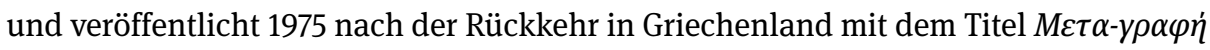

84 Brief von Klaus Schulz an Walter Höllerer vom 31. August 1966, Nachlass Walter Höllerer, Literaturarchiv Sulzbach-Rosenberg, Signatur 03WH/AB/12,37; vgl. dazu den Brief von Schulz vom 29. März 1966 (ebd., Signatur 03WH/AB/12,14) und den Brief von Sigfred Taubert an Höllerer vom 16. August 1966 (ebd., Signatur 03WH/AB/12,35).

85 Brief von Schulz an Höllerer vom 07. September 1967; ebd., Signatur 03WH/AB/13,5; vgl. auch die von Taubert an Höllerer weitergeleiteten alternativen Kostenanschläge für die Ausstellung.

86 Fleischer (Anm. 6), 263 mit Hinweis auf das Politische Archiv des Auswärtigen Amtes, Signatur B97/365, Kulturpolitischer Jahresbericht 1967, S. 12.

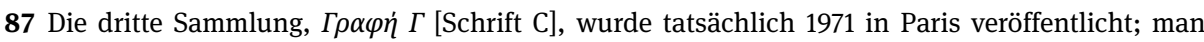
kann so davon ausgehen, dass Aravantinou während der Zeit des Künstlerprogramms an diesem Buch feilte. Einen Teil ihrer Berliner Zeit widmete sie jedoch der Untersuchung der Lage griechischer Gastarbeiter:innen und ihrer Stimmungen, der „Parias der Bahnhöfe“ als „Bewohner des Vereinigten Europas von morgen“, wie sie schrieb in: Mando Aravantinou: Die Europäer von morgen, in: François Bondy (Hrsg.): So sehen sie Deutschland. Staatsmänner, Schriftsteller und Publizisten aus Ost und West nehmen Stellung, Stuttgart 1970, 165-180, hier: 180. - Die Thematik der griechischen Migration bei der griechischsprachigen Berliner Weltliteratur wäre eine komplementäre Untersuchung wert; vgl. etwa neben den Erinnerungen von Aravantinou und Koumantareas die Erzählung von Vassilis Vassilikos „Die Arbeiter und die Sendung um 20 Uhr 20“, in deutscher Übersetzung in: Joachimides (Hrsg.): Athen in Berlin (Anm. 55), 74-75; und die Environments „Gastarbeiter - Fremdarbeiter“ von Vlassis Caniaris, siehe Endlich/Höynck (Hrsg.): Blickwechsel (Anm. 50), 164 und 386; sowie den Beitrag „Die Arbeitsmigration der 1960er und -70er Jahre im Spiegel griechischer Künstler in der Bundesrepublik Deutschland“ von Eleana Stoikou im Compendium der deutsch-griechischen Verflechtungen (https://comdeg.eu/compendium/). 
$\eta \dot{\eta} \varepsilon \mu \pi \varepsilon \iota \rho i \alpha$ бvvó $\rho \omega v$ [Nach-schrift oder Grenzerfahrung], ist eine prägende Erinnerung nicht zu übersehen, die augenblicklich die beiden zusammengebracht hatte.

Die Rede ist von einer Massenerschießung griechischer Zivilisten im März 1943 im besetzten Athen, deren Zeuge unerwartet sowohl Mando Aravantinou wurde als auch Walter Höllerer, dort eingesetzt als Funker in Kifissia. ${ }^{88}$ Beide, die griechische Dichterin und der ,junge Dichter als Soldat“, haben dieser Erfahrung jeweils ein Gedicht gewidmet, Aravantinou den sechsten Teil ihrer $\Gamma \rho \alpha \varphi \eta \dot{B}$ [Schrift B], Höllerer das Gedicht „Ich sah ich hörte“, anhand dessen er in seinem poetologischen Vortrag „Wie entsteht ein Gedicht“ das Programm und die Notwendigkeit des metamorphischen Gedichtes jenseits der Alternative poesie pure und poesie engagée exemplifiziert hatte.

In einer nicht datierten Karte an Höllerer, die die Typoskripte ihrer ins Französische übersetzten Sammlungen „Écriture A et B“ begleitete, verwies Aravantinou auf die diesbezügliche Stelle in ihrem Gedicht und erklärte die Transposition des gemeinsamen Erlebnisses in den Mai anstatt wie bei Höllerer im Monat März:

À l'occasion je vous envoie les deux manuscrits de l'Écriture A et B. La sixième partie de l'Écriture B concerne le problème que nous avions abordé hier.

La partie inclue outre les pages 36 jusqu’à 41, est la partie des mémoires de ce matin de Mars Mai - 1943.

M. A.

PS. Le mot Mai en grec a beaucoup plus de poids que Mars. C’est pour cela que j’ai employé le mot Mai. ${ }^{89}$

In ihrem Aufsatz „Die Europäer von morgen“ hat Aravantinou die Stelle aus Höllerers Essay „Wie entsteht ein Gedicht“ mit der überkreuzten Erinnerung an jenen Märzmorgen zitiert und die Verflechtung nicht als „Zufall“ sondern als „,seltene, unschätzbare Begegnung mit Büchern“ apostrophiert:

Die Erinnerung überspannt ein Vierteljahrhundert, sie schreitet unaufhaltsam rückwärts, ein Tag im März, Schatten und Blicke, ein bestimmtes Datum im Frühling, doch lasse ich besser jemand anderen erzählen:

„Ich spreche von einem Märzmorgen des Jahres 1943 zwischen 6 und 7 Uhr in Griechenland. Von einem LKW aus, der Ersatzteile holen sollte, beobachtete ich zufällig die Erschießung von ungefähr zwanzig griechischen Zivil-Geiseln auf einem Truppenübungsplatz unmittelbar vor dem Berg Hymettos bei Athen. Es war eine unheimliche Mechanik und maschinelle Lapidarität in diesem Ereignis. Es war wie ein marionettenhaftes Schattentheater, ein Bild, das sich, wie ich inzwischen gemerkt habe, jeder Metapher, jeder Beschreibung und jeder Tonart entzieht. [...]

Sah Pelztier sich ein Nest baun im Wurzelwerk,

Männer Papier verbrennen morgens,

88 Vgl. dazu Cryssoula Kambas: Junger Dichter als Soldat. Die Besatzung Griechenlands bei Walter Höllerer und Michael Guttenbrunner, in: Kambas/Mitsou (Hrsg.): Die Okkupation Griechenlands im zweiten Weltkrieg (Anm. 15), 421-451.

89 Nachlass Walter Höllerer, Literaturarchiv Sulzbach-Rosenberg, Signatur 03WH/CA/7,6. 
Sah Tote, ein Feld von Toten.“90

In ihrem Berliner Tagebuch hat Aravantinou, quasi um das poetologische Gespräch weiterzuführen, eine frühere Fassung aus „Ich sah ich hörte“ ins Griechische übersetzt, eine von jenen, die Höllerer als unlyrisch empfand, wo „das Motiv immer wieder auch als Motiv laut werden“ wollte, „und nicht nur als Anstoßkraft, als Motor für das Gedicht" wirkte; es ist als ob sie mit Höllerers poetologischer Kritik an folgende Verse als „epische Erlebnisberichte in Versform“ nicht einverstanden wäre und jenes „Selbstmitleid“ nicht so wie Höllerer als „störend“91 empfand:

,Sah Tote, ein Feld von Toten

Unter Stein und Asseln Ohrwurm Rollasseln Tausendfuß

Von Geiseln auf Schießplätzen, und ich

Blieb stumm.

Die Erinnerung wurde konkret, bekam eine Umgebung, eine Chronologie, wurde enthüllt, erzählte das Geschehen. Die Zeugenaussage wurde von zwei Personen, Höllerer und mir, an zwei verschiedenen Punkten der Stadt erlebt. Wir, stumme Randfiguren, lebten den gleichen Akt des Dramas, das ohne unsere Teilnahme gespielt wurde, jeder von uns von verschiedenen Seiten der Barrikade. Aber nein, die Zeugenaussage wurde von derselben Barrikade geschrieben, wo Augenblicke, Erinnerungen und Traumata unseren Affekt und unsere Gedanken besiegelten, diese, die das Zivil-Ich zu vergessen versucht. Die Worte passten damals und heute nicht, aber es gibt diese unerbittliche Erinnerung, die blutet, wo immer ich sie berühre. ${ }^{92}$

Wir haben uns die Frage gestellt, wie in der Perspektivierung der schweren Bürde der jüngsten bilateralen Vergangenheit die literarische Mobilität, der Literaturtransfer der neugriechischen Literatur in deutscher Sprache im geteilten Berlin, vorzustellen ist im Rahmen der kulturpolitischen Aktivitäten, die die Berliner 1960er Jahre in Ost und West geprägt haben. Unser Fazit: Seit den späten 1950er bis zu den späten 1960er

90 Aravantinou (Anm. 87), 170-171. Das Höllerer-Zitat, in: Walter Höllerer: Gedichte. Wie entsteht ein Gedicht, Frankfurt a. M. 1964, 72 und 65. Aravantinou zitiert lieber die achte Strophe des Gedichts im Gegensatz zu Höllerer, der nach dem Wort „entzieht“ schrieb: „Es ist versteckt im Auftakt des Gedichts: Ich sah ich hörte Reih’n, gebückt, Gesichter, / Und Pfiffe, Rufe - lass vorübergehn / und flog vorbei“ (ebd. 72-73).

91 Ebd. 73 und 74.

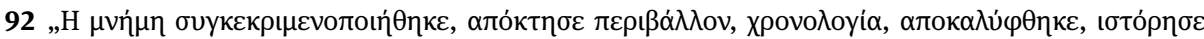

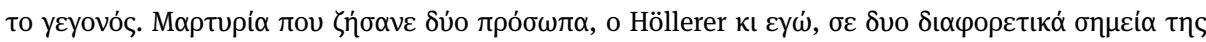

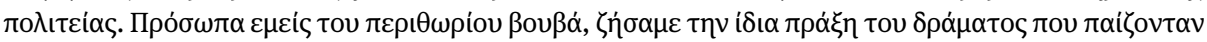

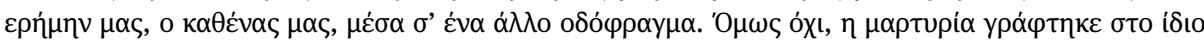

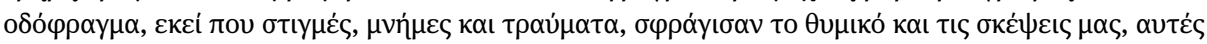

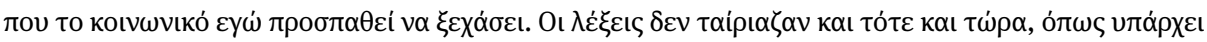
$\alpha$

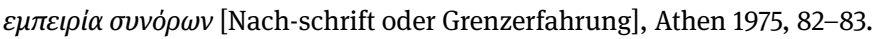


Jahren überkreuzten sich in Berlin, in unterschiedlichen Konstellationen der Ost- und Westberliner Kulturpolitik und Kulturoffensive, die Wege griechischer Künstler:innen und Autor:innen, die aus Griechenland und den Realitäten des „dreißigjährigen Krieges“ geflohen waren - ob Iannis Xenakis, Stipendiat des französischen Staates, mit den Wunden der Straßenschlachten vom Dezember 1944, ob Melpo Axioti, 1950 aus Paris ausgewiesen, und Dimitris Hadzis, 1952 in Griechenland in Abwesenheit zum Tode verurteilt, ob der staatenlose Bildhauer, Autor und Übersetzer Vagelis Tsakiridis, Vassilis Vassilikos, der von einer Reise 1967 nach Griechenland nicht zurückkehrte, oder Mando Aravantinou, die die Einladung als „artist in residence“ im Berliner Künstlerprogramm zum Anlass ihrer politischen Emigration machte. Als Teil der Berliner „Weltliteraturen“ hat die griechische Exilliteratur der Nachkriegszeit im deutschgriechischen Dreieck Wege der bilateralen Erinnerungskultur erprobt, Spielformen der Solidarität und der gegenseitigen Wahrnehmung ausgehandelt, und mit den in Berlin entstandenen Werken Die Ungeschützten, Mein Zuhause und Nach-Schrift oder Grenzerfahrung oder den in Berlin übersetzten Romanen $Z$ und Der Marsch der Neun sich auf die Suche nach einer neuen Tonlage für die zukünftige Literatur begeben.

Die geteilte Stadt des Kalten Krieges hat auch in diesem Fall als Horchposten, Versuchsbank oder Laboratorium gedient, wie es Michel Butor auf den Punkt gebracht hatte; Walter Höllerer hat es, bezogen auf den am 13. August 1961 begonnenen Mauerbau, so formuliert: „Dieses Datum hat die Verrenkungen, die an der Sprache in der Gegenwart vorgenommen werden, die Verdrehungen und Maskierungen von Begriffen und von Tonlagen noch einmal deutlich zusammengefasst.“93

93 Zitiert nach Michael Peter Hehl: Poetik der Institution. Walter Höllerers institutionelles Engagement und die Literatur der Moderne, in: kultuRRevolution 63 (2012), 45-53, hier: 47. 
